J. Brüdern and T. D. Wooley

Nagoya Math. J.

Vol. 163 (2001), 13-53

\title{
ON WARING'S PROBLEM: THREE CUBES AND A SIXTH POWER
}

\author{
JÖRG BRÜDERN AND TREVOR D. WOOLEY ${ }^{1}$
}

\begin{abstract}
We establish that almost all natural numbers not congruent to 5 modulo 9 are the sum of three cubes and a sixth power of natural numbers, and show, moreover, that the number of such representations is almost always of the expected order of magnitude. As a corollary, the number of representations of a large integer as the sum of six cubes and two sixth powers has the expected order of magnitude. Our results depend on a certain seventh moment of cubic Weyl sums restricted to minor arcs, the latest developments in the theory of exponential sums over smooth numbers, and recent technology for controlling the major arcs in the Hardy-Littlewood method, together with the use of a novel quasi-smooth set of integers.
\end{abstract}

\section{$\S 1$. Introduction}

It is widely expected that for any fixed positive integer $k$, all large natural numbers satisfying the necessary congruence conditions should be representable as the sum of three cubes and a $k$ th power of natural numbers. Let $\nu_{k}(n)$ denote the number of representations of the positive integer $n$ in this manner. Then a formal application of the circle method leads to the conjecture that the asymptotic formula

$$
\nu_{k}(n) \sim \Gamma\left(\frac{4}{3}\right)^{3} \mathfrak{S}_{k}(n) n^{1 / k}
$$

should hold, where

$$
\mathfrak{S}_{k}(n)=\sum_{q=1}^{\infty} q^{-4} \sum_{\substack{a=1 \\(a, q)=1}}^{q} \sum_{1 \leq x_{1}, \ldots, x_{4} \leq q} e\left(\frac{a}{q}\left(x_{1}^{3}+x_{2}^{3}+x_{3}^{3}+x_{4}^{k}-n\right)\right)
$$

denotes the singular series associated with the representation problem at hand. Here, and throughout, we write $e(z)$ for $e^{2 \pi i z}$. In this paper we show

Received January 12, 2000.

1991 Mathematics Subject Classification: 11P05, 11L15, 11P55.

${ }^{1}$ Packard Fellow, and supported in part by NSF grant DMS-9622773. The second author is grateful to the Mathematisches Institut A at Stuttgart for its generous hospitality during the period in which this paper was written. 
that $\nu_{6}(n)$ is almost always as large as is predicted by the heuristic asymptotic formula.

THEOREM 1. There are positive numbers $C$ and $\delta$ such that the inequality

$$
\nu_{6}(n) \geq C \mathfrak{S}_{6}(n) n^{1 / 6}
$$

fails to hold for at most $O\left(N^{1-\delta}\right)$ of the natural numbers $n$ not exceeding $N$.

It follows from the classical theory of singular series that $\mathfrak{S}_{6}(n) \gg 1$ uniformly in $n \not \equiv 5(\bmod 9)$ (see $\S 8$ below for a discussion of this issue), and so it follows from Theorem 1 that almost all such natural numbers $n$ are the sum of three cubes and a sixth power of natural numbers. We note that a consideration of congruence conditions modulo 9 reveals that $\nu_{6}(n)=0$ whenever $n \equiv 5(\bmod 9)$. Another immediate consequence of Theorem 1 is that all large natural numbers are the sum of six cubes and two sixth powers of natural numbers.

THEOREM 2. Let $R(n)$ denote the number of representations of the integer $n$ as the sum of six cubes and two sixth powers of natural numbers. Then for all sufficiently large $n$, one has $R(n) \gg n^{4 / 3}$.

This lower bound again coincides with the expected order of magnitude for $R(n)$. In order to deduce Theorem 2 from Theorem 1 , one merely notes that

$$
R(n)=\sum_{1 \leq m \leq n} \nu_{6}(m) \nu_{6}(n-m) .
$$

Since Theorem 1 shows that $\nu_{6}(m)$ and $\nu_{6}(n-m)$ are simultaneously $\gg n^{1 / 6}$ for all but $o(n)$ of the integers $m$ with

$$
m \neq \equiv 5(\bmod 9), \quad n-m \not \equiv 5(\bmod 9) \quad \text { and } \quad 1 \leq m \leq n,
$$

the lower bound recorded in Theorem 2 is immediate. Considerably weaker lower bounds for $R(n)$ have been claimed by Ming Gao Lu [13] and Breyer [1]. Both authors employ an asymmetric choice of generating functions within an application of the circle method, thereby excluding an almost all result for three cubes and a sixth power from the scope of their methods. The presence of large common factors between certain of the variables, 
moreover, necessitates that their arguments discard almost all representations from the discussion.

Our methods are also applicable when $k \leq 5$, and lead to conclusions allied to those of Theorems 1 and 2 which read mutatis mutandis, save that congruence conditions may be omitted. These results seem to be new when $k=5$, but for $k=4$ results of the type considered here are at least implicit in the literature. Brüdern [4] has shown that the number, $R^{*}(n)$, of representations of a large integer $n$ as the sum of six cubes and two fourth powers satisfies $R^{*}(n) \gg n^{3 / 2}$, a lower bound which corresponds to that recorded in Theorem 2 . The arguments of that paper are readily modified to establish the existence of positive numbers $c$ and $\delta$ with the property that $\nu_{4}(n)>c n^{1 / 4}$ for all but $O\left(N^{1-\delta}\right)$ of the integers $n$ with $1 \leq n \leq N$. Further work within this circle of ideas concerns the exceptional set $\mathcal{E}_{k}(X)$, which we define by

$$
\mathcal{E}_{k}(X)=\left\{1 \leq n \leq X: \nu_{k}(n)=0\right\}
$$

Brüdern [2] has provided bounds of the type $\operatorname{card}\left(\mathcal{E}_{k}(X)\right) \ll X^{1-\delta_{k}}$ with explicit values of $\delta_{k}$, for $k=4$ and 5 , and these estimates have subsequently been improved by Lu [14]. When $k=3$, meanwhile, these problems reduce to the classical Waring problem for four cubes. While this is certainly the most prominent member in this series of problems, describing its long history is hardly the point of the present paper. We refer the reader to Vaughan [17], Brüdern [5], Wooley [24] and Kawada [12] for an account of recent developments concerning this problem.

We establish Theorem 1 through the use of the Hardy-Littlewood method. The proof has many similarities with Vaughan's approach [18] to the seven cubes theorem, though we must work harder to achieve success. Indeed, our methods make fundamental use of a minor arc estimate involving a product of seven cubic exponential sums which is of independent interest; it has already found applications beyond those in this paper (part IV of Brüdern, Kawada and Wooley [7]). This paper is therefore organised in two chapters. The first deals with the minor arc estimate alluded to above, while the second is devoted to the proof of Theorem 1. Both chapters are equipped with an introductory section to which the reader is referred for a finer discussion of the underlying ideas. However, we do take this opportunity to draw the reader's attention to the use of a variant of the usual smooth Weyl sum in the second chapter, the point being that control of such exponential sums on the major arcs of the Hardy-Littlewood 
dissection is substantially enhanced. This device should be useful whenever smooth Weyl sums occur in an application of the Hardy-Littlewood method.

Throughout, $\varepsilon$ and $\eta$ will denote sufficiently small positive numbers. We usually take $P$ to be the basic parameter, a large real number depending at most on $\varepsilon$ and $\eta$. We use $\ll$ and $\gg$ to denote Vinogradov's well-known notation, implicit constants depending at most on $\varepsilon$ and $\eta$. Also, we write $[x]$ for the greatest integer not exceeding $x$, and $\|y\|$ for $\min _{n \in \mathbb{Z}}|y-n|$. In an effort to simplify our analysis, we adopt the following convention concerning the parameter $\varepsilon$. Whenever $\varepsilon$ appears in a statement, we assert that for each $\varepsilon>0$ the statement holds for sufficiently large values of the main parameter. Note that the "value" of $\varepsilon$ may consequently change from statement to statement, and hence also the dependence of implicit constants on $\varepsilon$.

The authors thank the referee for careful comments.

\section{A Minor Arc Estimate related to Seven Cubes}

\section{$\S 2$. Introductory comments}

This chapter, which is self-contained, establishes a technical minor arc estimate to which we have referred in the introduction, and which we expect to be useful elsewhere. It is necessary to fix some notation before the key result can be described. We define the classical Weyl sums $f(\alpha)=f(\alpha ; P)$ and $F(\alpha)=F(\alpha ; P)$ by

$$
f(\alpha ; P)=\sum_{P<x \leq 2 P} e\left(\alpha x^{3}\right) \quad \text { and } \quad F(\alpha ; P)=\sum_{1 \leq x \leq P} e\left(\alpha x^{3}\right),
$$

and when $\mathcal{B} \subseteq[1, P] \cap \mathbb{Z}$, we define the exponential sum $h(\alpha)=h(\alpha ; \mathcal{B})$ by

$$
h(\alpha ; \mathcal{B})=\sum_{x \in \mathcal{B}} e\left(\alpha x^{3}\right) .
$$

In the applications within this paper, and in work of Brüdern, Kawada and Wooley [7], we restrict $\mathcal{B}$ to be one of two sets of smooth numbers. In this context, when $R$ and $Q$ are real numbers with $1 \leq R \leq Q$, define the sets of smooth numbers $\mathcal{A}(Q, R)$ and $\mathcal{A}^{*}(Q, R)$ by

$$
\begin{gathered}
\mathcal{A}(Q, R)=\{n \in[1, Q] \cap \mathbb{Z}: p \text { prime, } p \mid n \Rightarrow p \leq R\}, \\
\mathcal{A}^{*}(Q, R)=\{n \in[1, Q] \cap \mathbb{Z}: p \text { prime, } p \mid n \Rightarrow \sqrt{R}<p \leq R\},
\end{gathered}
$$


and then define the subset $\mathcal{C}(P, R)$ of $\mathcal{A}(P, R)$ by

(2.4) $\mathcal{C}(P, R)$

$$
=\bigcup_{k=0}^{K}\left\{l m: m \in \mathcal{A}^{*}\left(2^{k} P / \sqrt{R}, R\right) \text { and } 2^{-1-k} \sqrt{R}<l \leq 2^{-k} \sqrt{R}\right\} \text {, }
$$

where $K=\left[\frac{1}{4} \log R\right]$. We remark that whenever $n \in \mathcal{C}(P, R)$, then $n$ is uniquely represented in the shape $n=l m$ with $m \in \mathcal{A}^{*}\left(2^{k} P / \sqrt{R}, R\right)$, $2^{-1-k} \sqrt{R}<l \leq 2^{-k} \sqrt{R}$ and $0 \leq k \leq K$, as is apparent by considering the prime factorisation of $n$. We confine attention to the situation in which $\mathcal{B}$ is equal either to $\mathcal{A}(P, R)$ or $\mathcal{C}(P, R)$. The mean value estimates which play an important role in our subsequent deliberations are recorded in the following lemma.

LEMma 1. Let $\xi$ denote the positive root of the polynomial $\xi^{3}+16 \xi^{2}+$ $28 \xi-8$, and put $\gamma=3 \xi /(8+2 \xi)$. Then for each $\varepsilon>0$, there exists a positive number $\eta_{0}=\eta_{0}(\varepsilon)$ such that whenever $R \leq P^{\eta_{0}}$ and $\mathcal{B} \subseteq \mathcal{A}(P, R)$, one has

$$
\int_{0}^{1}\left|F(\alpha ; 2 P)^{2} h(\alpha ; \mathcal{B})^{4}\right| d \alpha \ll P^{3+\xi+\varepsilon}
$$

and

$$
\int_{0}^{1}|h(\alpha ; \mathcal{B})|^{5} d \alpha \ll P^{\frac{5}{2}+\gamma+\varepsilon}
$$

Proof. By hypothesis one has $\mathcal{B} \subseteq \mathcal{A}(P, R)$, so that on considering the underlying diophantine equations and noting (2.1) and (2.2), the estimate (2.5) is immediate from Theorem 1.2 of Wooley [24]. The upper bound (2.6), meanwhile, follows from Lemma 5.1 of Wooley [24] in like manner, since the fifth moment estimate provided by the latter rests, ultimately, on the number of solutions of certain underlying diophantine equations, and such is not increased by substituting for the range of the variables $\mathcal{B}$ in place of $\mathcal{A}(P, R)$.

We note for future reference that the numbers $\xi$ and $\gamma$ occurring in the statement of Lemma 1 satisfy the inequalities

$$
0.2495681<\xi<0.2495682 \text { and } 0.0880918<\gamma<0.0880919
$$


Finally, we take $Y$ to be a real number satisfying $1 \leq Y \leq P^{1 / 7}$ to be fixed later, and write $Q=P / Y$. When $\mathcal{B} \subseteq[1, Q] \cap \mathbb{Z}$, we then define the exponential sum $g(\alpha)=g(\alpha ; Y ; \mathcal{B})$ by

$$
g(\alpha ; Y ; \mathcal{B})=\sum_{\substack{Y<p \leq 2 Y \\ p \equiv 2(\bmod 3)}} h\left(\alpha p^{3} ; \mathcal{B}\right),
$$

in which the summation is over prime numbers $p$.

We are now equipped to announce the main result of this chapter.

THEOREM 3. Suppose that $Y$ is a real number with $P^{1 / 8} \leq Y \leq P^{1 / 7}$. Let $\mathfrak{m}$ denote the set of real numbers $\alpha \in[0,1]$ such that whenever $q$ is a natural number with $\|q \alpha\| \leq Y^{3} P^{-2}$, then one has $q \geq P Y^{3}$. Also, when $\mathcal{B} \subseteq \mathcal{A}(Q, R)$, define the mean value $I(Y)=I(Y ; \mathcal{B})$ by

$$
I(Y)=\int_{\mathfrak{m}}\left|f(\alpha ; P)^{2} g(\alpha ; Y ; \mathcal{B})^{5}\right| d \alpha .
$$

Then for each $\varepsilon>0$, there exists a positive number $\eta_{0}=\eta_{0}(\varepsilon)$ such that whenever $R \leq P^{\eta_{0}}$, one has

$$
I(Y) \ll P^{4+\gamma+\varepsilon} Y^{-1-\gamma}
$$

A weaker estimate for $I(Y)$ occurs inter alia in work of Vaughan [18], with $Y$ restricted to the immediate vicinity of $P^{1 / 8}$. The argument of Vaughan yields the upper bound $I\left(P^{1 / 8}\right) \ll P^{4-\frac{1}{32}+\varepsilon}$, whereas Theorem 3 establishes an estimate at least as strong as

$$
I(Y) \ll P^{3.9521}
$$

in the whole range of $Y$. The bound provided by Theorem 3 is of course strongest when $Y=P^{1 / 7}$, in which case the exponent in (2.9) reduces to 3.9327. However, larger values of $Y$ thin out the minor arcs, and this is a nuisance in applications, due to increasing difficulties in handling error terms in the treatment of the major arcs. In practice one must balance $I(Y)$ against the latter error terms, and thus the optimal choice of $Y$ may be smaller than $P^{1 / 7}$. In the applications within this paper, only values of $Y$ close to $P^{1 / 8}$ will be employed, but it is hoped that the superior bounds available for larger $Y$ will be of utility elsewhere. 
The improvements over the aforementioned work of Vaughan [18] represented in Theorem 3 stem from three sources which are best described within a sketch of the proof of Theorem 3 . We begin with a $p$-adic iteration restricted to minor arcs. This procedure is due to Vaughan [17]. It transforms $|f(\alpha)|^{2}$ into a quadratic exponential sum, and also brings into the analysis fifth moments of $|h(\alpha ; \mathcal{B})|$. We estimate the latter through the "breaking convexity" device of Wooley [24], a tool unavailable at the time of writing of [18]. This already provides some savings. In estimating the quadratic exponential sum resulting from differencing $|f(\alpha)|^{2}$, we make further savings by averaging over $p$. This idea is not new; the work of $\S \S 5-7$ of Vaughan [17] contains all of the necessary ideas. We collect together the relevant details in an abstracted form in Lemma 4 below. Finally, the wider available range for $Y$ is a consequence of a more sophisticated removal of certain undesirable common factors which arise in the variables underlying the $p$-adic iteration. It is the latter which entails the elaborate analysis described in $\S 5$ below.

\section{$\S 3$. Some auxiliary equations and inequalities}

We begin our approach to Theorem 3 by collecting together various mean value estimates for cubic exponential sums. For the remainder of this chapter we write, for the sake of concision,

$$
f(\alpha)=f(\alpha ; P), \quad g(\alpha)=g(\alpha ; Y ; \mathcal{B}), \quad h(\alpha)=h(\alpha ; \mathcal{B}),
$$

and suppose throughout that $\mathcal{B} \subseteq \mathcal{A}(Q, R)$. Also, we suppose that $\eta_{0}$ is a positive number sufficiently small in the context of Lemma 1 , and take $R$ to be a real number satisfying $R \leq P^{\eta_{0} / 10}$.

LEMmA 2. Whenever $P^{1 / 8} \leq Y \leq P^{1 / 7}$, one has

$$
\int_{0}^{1}\left|F(\alpha ; 2 P)^{2} g(\alpha)^{4}\right| d \alpha \ll P^{3+\varepsilon} Y^{2} .
$$

Proof. The special cases $Y=P^{1 / 8}$ and $Y=P^{1 / 7}$ are contained in all essentials in Lemma 1 of Vaughan [18] and the Proposition of Brüdern [6], respectively, but there is no explicit reference for intermediate values of $Y$. Although it would be straightforward to extend the argument underlying the proof of Lemma 1 of Vaughan [18], for the sake of completeness we give a proof which is somewhat simpler than Vaughan's original treatment. Some 
of the ideas introduced here will also prove profitable in the verification of the next lemma.

Let $\rho(n)$ denote the number of integral solutions of the equation

$$
n=x^{3}+p_{1}^{3} y_{1}^{3}+p_{2}^{3} y_{2}^{3},
$$

with

$$
(3.2) \quad 1 \leq x \leq 2 P, \quad y_{i} \in \mathcal{B}, \quad Y<p_{i} \leq 2 Y, \quad p_{i} \equiv 2(\bmod 3) \quad(i=1,2)
$$

Also, let $\rho_{1}(n)$ denote the corresponding number of solutions in which the additional condition $\left(x, p_{1} p_{2}\right)=1$ holds. Observe that in any solution $x, \mathbf{p}, \mathbf{y}$ of (3.1) counted by $\rho(n)$ but not counted by $\rho_{1}(n)$, one has that $p_{1} \mid x$ or $p_{2} \mid x$. Let $\rho_{2}(n)$ denote the number of solutions of (3.1) satisfying (3.2) and the conditions $p_{1}=p_{2}$ and $p_{1} \mid x$, and let $\rho_{3}(n)$ denote the corresponding number of solutions in which $p_{1} \neq p_{2}$ and $p_{1} \mid x$. Then by symmetry one has

$$
\rho(n) \leq \rho_{1}(n)+\rho_{2}(n)+2 \rho_{3}(n) .
$$

On making use of Cauchy's inequality and considering the underlying diophantine equation, we therefore deduce that

$$
\int_{0}^{1}\left|F(\alpha ; 2 P)^{2} g(\alpha)^{4}\right| d \alpha=\sum_{n \in \mathbb{N}} \rho(n)^{2} \ll S_{1}+S_{2}+S_{3}
$$

where

$$
S_{j}=\sum_{n \in \mathbb{N}} \rho_{j}(n)^{2} \quad(j=1,2,3) .
$$

We first estimate $S_{2}$. Observe that if $x, \mathbf{p}, \mathbf{y}$ is any solution of (3.1) counted by $\rho_{2}(n)$, then we have $p_{1} \mid x$, and thus we may substitute $x=p_{1} z$. In this way we deduce that

$$
\rho_{2}(n) \leq \sum_{Y<p \leq 2 Y} \sigma_{2}(n, p)
$$

where $\sigma_{2}(n, p)$ denotes the number of integral solutions of the equation

$$
n=p^{3}\left(z^{3}+y_{1}^{3}+y_{2}^{3}\right),
$$

with

$$
1 \leq z \leq 2 Q \quad \text { and } \quad y_{i} \in \mathcal{B} \quad(i=1,2)
$$


By Cauchy's inequality, therefore, on considering the underlying diophantine equation, we derive from (3.4) the estimate

$$
\begin{aligned}
S_{2} & \leq Y \sum_{Y<p \leq 2 Y} \sum_{n \in \mathbb{N}} \sigma_{2}(n, p)^{2} \\
& \leq Y^{2} \int_{0}^{1}\left|F(\alpha ; 2 Q)^{2} h(\alpha)^{4}\right| d \alpha .
\end{aligned}
$$

The estimate (2.5) of Lemma 1 therefore yields

$$
S_{2} \ll Q^{3+\xi+\varepsilon} Y^{2}
$$

Next consider $S_{3}$. When $x, \mathbf{p}, \mathbf{y}$ is a solution of (3.1) counted by $\rho_{3}(n)$, we may again substitute $x=p_{1} z$. If $w$ is an integer of the shape $w=$ $p_{2} y_{2}$, with $p_{2}$ and $y_{2}$ as in (3.2), moreover, the latter representation of $w$ is necessarily unique, since $\left(p_{2}, y_{2}\right)=1$. Also, since $p_{1} \neq p_{2}$, one has that $\left(p_{1}, p_{2} y_{2}\right)=1$. Thus we deduce that

$$
\rho_{3}(n) \leq \sum_{Y<p \leq 2 Y} \sigma_{3}(n, p),
$$

where $\sigma_{3}(n, p)$ denotes the number of integral solutions of the equation

$$
n=p^{3}\left(z^{3}+y^{3}\right)+w^{3},
$$

with

$$
1 \leq w \leq 2 P, \quad(w, p)=1, \quad 1 \leq z \leq 2 Q, \quad y \in \mathcal{B}
$$

By Cauchy's inequality, it follows from (3.4) that

$$
S_{3} \ll Y \sum_{Y<p \leq 2 Y} \sum_{n \in \mathbb{N}} \sigma_{3}(n, p)^{2},
$$

whence $S_{3} \ll Y S_{4}$, where $S_{4}$ denotes the number of integral solutions of the equation

$$
w_{1}^{3}-w_{2}^{3}=p^{3}\left(z_{1}^{3}-z_{2}^{3}+y_{1}^{3}-y_{2}^{3}\right),
$$

with $Y<p \leq 2 Y$ and

$$
1 \leq w_{i} \leq 2 P, \quad\left(w_{i}, p\right)=1, \quad 1 \leq z_{i} \leq 2 Q, \quad y_{i} \in \mathcal{B} \quad(i=1,2) .
$$

Since $Y \leq P^{1 / 7}$, it follows from Theorem A of Vaughan [17] that

$$
S_{4} \ll P^{\frac{3}{2}+\varepsilon} Q^{2} Y^{-\frac{5}{2}},
$$


whence

$$
S_{3} \ll P^{\frac{3}{2}+\varepsilon} Q^{2} Y^{-\frac{3}{2}}
$$

It remains to estimate $S_{1}$. Define the exponential sum $F_{d}(\alpha)$ by

$$
F_{d}(\alpha)=\sum_{\substack{1 \leq x \leq 2 P \\(x, d)=1}} e\left(\alpha x^{3}\right)
$$

Then on considering the underlying diophantine equation, it follows from (3.4) that

$$
S_{1}=\int_{0}^{1}\left|\sum_{p_{1}, p_{2}} F_{p_{1} p_{2}}(\alpha) h\left(\alpha p_{1}^{3}\right) h\left(\alpha p_{2}^{3}\right)\right|^{2} d \alpha
$$

where the summation over $p_{1}$ and $p_{2}$ is subject to (3.2). Two applications of Cauchy's inequality now yield

$$
S_{1} \ll Y^{2} \sum_{p_{1}, p_{2}} \int_{0}^{1}\left|F_{p_{1} p_{2}}(\alpha)^{2} h\left(\alpha p_{1}^{3}\right)^{4}\right| d \alpha .
$$

Consequently, on considering the underlying diophantine equation, we deduce that

$$
S_{1} \ll Y^{3} S_{5},
$$

where $S_{5}$ denotes the number of integral solutions of the equation

$$
x_{1}^{3}-x_{2}^{3}=p_{1}^{3}\left(y_{1}^{3}-y_{2}^{3}+y_{3}^{3}-y_{4}^{3}\right),
$$

with $p_{1}$ subject to $(3.2)$ and $y_{j} \in \mathcal{B}(1 \leq j \leq 4)$, and also

$$
1 \leq x_{i} \leq 2 P, \quad\left(x_{i}, p_{1}\right)=1 \quad(i=1,2)
$$

But on combining Lemma 3.7 of Vaughan [19] with Lemma 1 above, one readily confirms that

$$
S_{5} \ll P^{3+\varepsilon} Y^{-1}+P^{\frac{10}{3}+\varepsilon} Y^{-\frac{11}{3}},
$$

and so we may conclude from (3.8) that

$$
S_{1} \ll P^{3+\varepsilon} Y^{2}+P^{\frac{10}{3}+\varepsilon} Y^{-\frac{2}{3}}
$$


On combining the conclusions of (3.3), (3.5), (3.6) and (3.9), we finally discover that for $P^{1 / 8} \leq Y \leq P^{1 / 7}$, one has the estimate

$$
\begin{aligned}
\int_{0}^{1} \mid F(\alpha ; & 2 P)^{2} g(\alpha)^{4} \mid d \alpha \\
& \ll Q^{3+\xi+\varepsilon} Y^{2}+P^{\frac{3}{2}+\varepsilon} Q^{2} Y^{-\frac{3}{2}}+P^{3+\varepsilon} Y^{2}+P^{\frac{10}{3}+\varepsilon} Y^{-\frac{2}{3}} \\
& \ll P^{3+\varepsilon} Y^{2},
\end{aligned}
$$

and hence the proof of the lemma is complete.

LEMma 3. Denote by $T(Y)$ the number of integral solutions of the equation

$$
p_{1}^{3}\left(x_{1}^{3}-x_{2}^{3}\right)=p_{2}^{3}\left(y_{1}^{3}-y_{2}^{3}+y_{3}^{3}-y_{4}^{3}\right)
$$

with

$$
\begin{gathered}
Y<p_{i} \leq 2 Y \quad \text { and } \quad 1 \leq x_{i} \leq 2 Q \quad(i=1,2) \\
y_{j} \in \mathcal{B} \quad(1 \leq j \leq 4)
\end{gathered}
$$

Then whenever $P^{1 / 8} \leq Y \leq P^{1 / 7}$, one has

$$
T(Y) \ll Y Q^{3+\xi+\varepsilon}
$$

Proof. Let $T_{0}(Y)$ denote the number of solutions of (3.10) counted by $T(Y)$ in which $p_{1}=p_{2}$, let $T_{1}(Y)$ denote the corresponding number of solutions with $p_{1} \neq p_{2}$ and $p_{2} \nmid x_{1} x_{2}$, and let $T_{2}(Y)$ denote the corresponding number of solutions with $p_{1} \neq p_{2}$ and $p_{2} \mid x_{1} x_{2}$. Then plainly,

$$
T(Y)=T_{0}(Y)+T_{1}(Y)+T_{2}(Y)
$$

We begin by estimating $T_{0}(Y)$. Suppose that $\mathbf{p}, \mathbf{x}, \mathbf{y}$ is a solution of (3.10) counted by $T_{0}(Y)$. Then since $p_{1}=p_{2}$, the number of available choices for $\mathbf{p}$ is $O(Y)$. Fixing any one such choice for $\mathbf{p}$, the equation (3.10) takes the shape

$$
x_{1}^{3}-x_{2}^{3}=y_{1}^{3}-y_{2}^{3}+y_{3}^{3}-y_{4}^{3},
$$

whence by (3.11) and (3.12), the estimate

$$
T_{0}(Y) \ll Y Q^{3+\xi+\varepsilon}
$$

is immediate from Lemma 1, on considering the underlying diophantine equation. 
Next suppose that $\mathbf{p}, \mathbf{x}, \mathbf{y}$ is a solution of (3.10) counted by $T_{2}(Y)$. Then since $p_{1} \neq p_{2}$ it follows from (3.10) that $p_{2} \mid x_{1}$ if and only if $p_{2} \mid x_{2}$. We may therefore substitute $x_{j}=p_{2} w_{j}(j=1,2)$, and thus deduce from (3.10) that

$$
p_{1}^{3}\left(w_{1}^{3}-w_{2}^{3}\right)=y_{1}^{3}-y_{2}^{3}+y_{3}^{3}-y_{4}^{3},
$$

with $w_{1}$ and $w_{2}$ integers satisfying $1 \leq w_{j} \leq 2 Q Y^{-1}(j=1,2)$. On noting that the number of representations of an integer $z$ in the shape $p w$, with $Y<p \leq 2 Y$ and $1 \leq w \leq 2 Q Y^{-1}$, is at most 8 , we arrive at the estimate

$$
T_{2}(Y) \ll Y T_{3}(Y)
$$

where $T_{3}(Y)$ denotes the number of integral solutions of the equation

$$
z_{1}^{3}-z_{2}^{3}=y_{1}^{3}-y_{2}^{3}+y_{3}^{3}-y_{4}^{3},
$$

with $\mathbf{y}$ satisfying (3.12), and $1 \leq z_{j} \leq 4 Q(j=1,2)$. Consequently, on considering the underlying diophantine equation, it follows from Lemma 1 that $T_{3}(Y) \ll Q^{3+\xi+\varepsilon}$, whence

$$
T_{2}(Y) \ll Y Q^{3+\xi+\varepsilon} .
$$

Finally, suppose that $\mathbf{p}, \mathbf{x}, \mathbf{y}$ is a solution of (3.10) counted by $T_{1}(Y)$. We substitute $u_{i}=p_{1} x_{i}(i=1,2)$, and observe that since $p_{1} \neq p_{2}$, one has $p_{2} \nmid u_{1} u_{2}$. Thus we deduce that $T_{1}(Y) \ll T_{4}(Y)$, where $T_{4}(Y)$ denotes the number of integral solutions of the equation

$$
u_{1}^{3}-u_{2}^{3}=p_{2}^{3}\left(y_{1}^{3}-y_{2}^{3}+y_{3}^{3}-y_{4}^{3}\right)
$$

with

$$
\begin{gathered}
Y<p_{2} \leq 2 Y, \quad 1 \leq y_{i} \leq Q \quad(1 \leq i \leq 4) \\
1 \leq u_{j} \leq 4 P, \quad\left(u_{j}, p_{2}\right)=1 \quad(j=1,2)
\end{gathered}
$$

But Theorem A of Vaughan [17] provides the estimate $T_{4}(Y) \ll P^{\frac{7}{2}+\varepsilon} Y^{-\frac{9}{2}}$, and thus

$$
T_{1}(Y) \ll P^{\frac{7}{2}+\varepsilon} Y^{-\frac{9}{2}}
$$

On collecting together (3.13)-(3.16), we find that for $P^{1 / 8} \leq Y \leq P^{1 / 7}$, one has

$$
T(Y) \ll Y Q^{3+\xi+\varepsilon},
$$

and thus the proof of the lemma is complete. 


\section{$\S 4$. Differencing restricted to minor arcs}

In this section we provide an abstracted version of an iterative method restricted to minor arcs, in the shape of Lemma 4 below. Hopefully, the main ideas of Vaughan $[17, \S \S 5-8]$ will be more easily accessible in this form. In advance of the statement of Lemma 4, we recall the definition of the minor arcs $\mathfrak{m}$ from the statement of Theorem 3 , and define the exponential sum $f_{d}(\alpha)$ similar to that defined in (3.7) by

$$
f_{d}(\alpha)=\sum_{\substack{P<x \leq 2 P \\(x, d)=1}} e\left(\alpha x^{3}\right) .
$$

Lemma 4. Suppose that $Y$ is a real number with $P^{1 / 8} \leq Y \leq P^{1 / 7}$, and let $S: \mathbb{R} \longrightarrow[0, \infty)$ be a Riemann integrable function of period 1 . Then

$$
\int_{\mathfrak{m}} \sum_{\substack{Y<p \leq 2 Y \\ p \equiv 2(\bmod 3)}}\left|f_{p}(\alpha)\right|^{2} S\left(p^{3} \alpha\right) d \alpha \ll P^{3 / 2+\varepsilon} Y^{-5 / 2} \int_{0}^{1} S(\alpha) d \alpha .
$$

Proof. In order to establish the lemma we must rework Lemmata 6,8 and 10 of Vaughan [17] with some care. Let $\mathfrak{n}$ denote the set of $\alpha \in[0,1)$ with the property that whenever $q$ is a natural number with $\|q \alpha\| \leq P Q^{-3}$, then one has $q>P$. By following the argument of the proof of Lemma 10 of Vaughan [17], beginning at equation (8.3) of that paper, we deduce that

$$
\int_{\mathfrak{m}} \sum_{\substack{Y<p \leq 2 Y \\ p \equiv 2(\bmod 3)}}\left|f_{p}(\alpha)\right|^{2} S\left(p^{3} \alpha\right) d \alpha \ll \mathfrak{I}
$$

where

$$
\mathfrak{I}=\int_{\mathfrak{n}} \sum_{\substack{Y<p \leq 2 Y \\ p \equiv 2(\bmod 3)}} \Phi_{p}(\alpha) S(\alpha) d \alpha
$$

and

$$
\Phi_{p}(\alpha)=\sum_{\substack{P<y \leq 2 P \\(y, p)=1}} 1+2 \Re\left(\sum_{\substack{1 \leq h \leq H \\ 2 P+h p^{3}<y \leq 4 P-h p^{3} \\(y, p)=1 \\ y \equiv h(\bmod 2)}} e\left(\frac{1}{4} \alpha h\left(3 y^{2}+h^{2} p^{6}\right)\right)\right),
$$

with $H=P Y^{-3}$. 
In the next step, we remove the coprimality condition $(y, p)=1$ from the definition of $\Phi_{p}(\alpha)$. This we achieve by following the argument of $\S 5$ of Vaughan [17]. For ease of comparison, we make use of the notation of [17], but introduce as little of this as is possible. Write

$$
\begin{gathered}
F(\beta, \gamma ; h)=\sum_{\substack{2 P<y \leq 4 P \\
y \equiv h(\bmod 2)}} e\left(\frac{3}{4} \beta y^{2}-\gamma y\right), \\
G_{h}(\rho, \sigma)=\sum_{\substack{Y<p \leq \min \left\{2 Y,(P /(2 h))^{1 / 3}\right\} \\
p \equiv 2(\bmod 3)}} e\left(\frac{1}{4} \rho p^{6}+\sigma p^{3}\right), \\
\Xi_{p}(\alpha)=2 \Re\left(\sum_{1 \leq h \leq H} e\left(\frac{1}{4} \alpha h^{3} p^{6}\right) \sum_{2 P p^{-1}+h p^{2}<y \leq 4 P p^{-1}-h p^{2}} e\left(\frac{3}{4} \alpha h p^{2} y^{2}\right)\right),
\end{gathered}
$$

and then define

$$
\begin{gathered}
T_{2}(p)=\int_{\mathfrak{n}} \Xi_{p}(\alpha) S(\alpha) d \alpha, \quad T_{3}=\int_{0}^{1} S(\alpha) d \alpha \\
T_{5}(\gamma, \theta)=\int_{\mathfrak{n}} \sum_{1 \leq h \leq H}\left|F(\alpha h, \gamma ; h) G_{h}\left(\alpha h^{3}, \theta \gamma h\right)\right| S(\alpha) d \alpha .
\end{gathered}
$$

On recalling (4.3), the proof of Lemma 6 of Vaughan [17] (see, in particular, equations (5.13) and (5.26) of [17]) shows that

$$
\mathfrak{I} \ll \mathfrak{I}_{1}+\mathfrak{I}_{2}+\left|\mathfrak{I}_{3}\right|
$$

where

$$
\text { (4.7) } \mathfrak{I}_{1}=P Y T_{3}, \quad \mathfrak{I}_{2}=(\log P) \sup _{\substack{0 \leq \gamma \leq 1 \\ \theta= \pm 1}} T_{5}(\gamma, \theta), \quad \mathfrak{I}_{3}=\sum_{\substack{Y<p \leq 2 Y \\ p \equiv 2(\bmod 3)}} T_{2}(p) .
$$

We investigate the terms $\mathfrak{I}_{j}(j=1,2,3)$ in turn. Observe first that when $Y \leq P^{1 / 7}$, one has $P Y \leq P^{3 / 2} Y^{-5 / 2}$, and thus when $i=1$ it follows from (4.4) and (4.7) that

$$
\mathfrak{I}_{i} \ll P^{3 / 2+\varepsilon} Y^{-5 / 2} \int_{0}^{1} S(\alpha) d \alpha
$$


In order to bound $\mathfrak{I}_{2}$, we begin by noting that as a consequence of Lemma 7 of Vaughan [17], the estimate

$$
\sup _{\alpha \in \mathfrak{n}}\left(\sum_{1 \leq h \leq H}|F(\alpha h, \gamma ; h)|^{2}\right) \ll H P^{1+\varepsilon}
$$

holds uniformly in $\gamma$. Furthermore, since $H^{3 / 4} Y^{2} \ll H Y$ in the range of $Y$ under consideration, a simplified version of the argument used to establish Lemma 8 of Vaughan [17] yields the bound

$$
\sup _{\alpha \in \mathfrak{n}}\left(\sum_{1 \leq h \leq H}\left|G_{h}\left(\alpha h^{3}, \pm \gamma h\right)\right|^{2}\right) \ll P^{\varepsilon} H Y,
$$

uniformly in $\gamma$. Consequently, on recalling (4.4), an application of Cauchy's inequality to (4.5) shows that

$$
T_{5}(\gamma, \theta) \ll H(P Y)^{1 / 2+\varepsilon} T_{3} \ll P^{3 / 2+\varepsilon} Y^{-5 / 2} T_{3},
$$

uniformly in $\gamma \in \mathbb{R}$ and $\theta= \pm 1$. It therefore follows from (4.7) that the inequality (4.8) holds with $i=2$.

Finally, suppose that $\alpha \in \mathfrak{n}$. Then an inspection of the argument on pages 155 and 156 of Vaughan [17] reveals that in the range of $Y$ under consideration, one has

$$
\sum_{\substack{Y<p \leq 2 Y \\ p \equiv 2(\bmod 3)}}\left|\Xi_{p}(\alpha)\right| \ll P^{3 / 2+\varepsilon} Y^{-5 / 2},
$$

except possibly when there is a natural number $q$ with

$$
\|q \alpha\| \leq\left(H^{2} Q^{2} P^{-2}\right) Q^{-3} \text { and } q \leq H^{2} Q^{2} P^{-2} .
$$

But since $P \leq Y^{8}$, the latter inequalities imply that $\|q \alpha\| \leq P Q^{-3}$ and $q \leq P$, contradicting our hypothesis that $\alpha \in \mathfrak{n}$. On recalling (4.4) and (4.7), we conclude from (4.9) that the inequality (4.8) holds with $i=3$.

The proof of the lemma is completed by collecting together (4.2), (4.6) and the estimates (4.8) for $1 \leq i \leq 3$.

In this paper we require only the case $S(\alpha)=|h(\alpha ; \mathcal{B})|^{5}$ of Lemma 4. We note for future reference that on combining Lemmata 1 and 4 , one obtains the estimate

$$
\sum_{\substack{Y<p \leq 2 Y \\ p \equiv 2(\bmod 3)}} \int_{\mathfrak{m}}\left|f_{p}(\alpha)^{2} h\left(p^{3} \alpha ; \mathcal{B}\right)^{5}\right| d \alpha \ll P^{\frac{3}{2}} Y^{-\frac{5}{2}} Q^{\frac{5}{2}+\gamma+\varepsilon} .
$$




\section{$\S 5$. The proof of Theorem 3}

Having marshalled our forces in the preceding sections, we may now seize the proof of Theorem 3. Recalling the definitions (2.1) and (4.1), we observe first that

$$
f(\alpha)=f_{p}(\alpha)+f\left(\alpha p^{3} ; P / p\right) .
$$

Thus it follows from (2.7) that

$$
f(\alpha) g(\alpha)=\sum_{p}\left(f_{p}(\alpha)+f\left(\alpha p^{3} ; P / p\right)\right) h\left(\alpha p^{3}\right),
$$

where here, and in the remainder of this section, the summation over primes $p$ and any allied variables is over the range $Y<p \leq 2 Y$ with $p \equiv 2(\bmod 3)$. In view of (2.8), one deduces from (5.2) that

$$
I(Y) \leq I_{1}+I_{2}
$$

where

$$
I_{1}=\int_{\mathfrak{m}}\left|\sum_{p} f_{p}(\alpha) h\left(\alpha p^{3}\right)\right|\left|f(\alpha) g(\alpha)^{4}\right| d \alpha
$$

and

$$
I_{2}=\int_{\mathfrak{m}}\left|\sum_{p} f\left(\alpha p^{3} ; P / p\right) h\left(\alpha p^{3}\right)\right|\left|f(\alpha) g(\alpha)^{4}\right| d \alpha .
$$

Suppose first that $I_{2} \geq I_{1}$. Then on recalling (2.8), it follows from an application of Hölder's inequality to (5.5) that

$$
I_{2} \leq I(Y)^{1 / 2} J_{1}^{1 / 4} J_{2}^{1 / 4}
$$

where

$$
J_{1}=\int_{0}^{1}|g(\alpha)|^{6} d \alpha \quad \text { and } \quad J_{2}=\int_{\mathfrak{m}}\left|\sum_{p} f\left(\alpha p^{3} ; P / p\right) h\left(\alpha p^{3}\right)\right|^{4} d \alpha
$$

But on considering the underlying diophantine equations, we deduce from Lemma 2 that

$$
J_{1} \ll P^{3+\varepsilon} Y^{2}
$$

whence by (5.3) and (5.6), one has

$$
I(Y) \ll P^{\frac{3}{2}+\varepsilon} Y J_{2}^{1 / 2} .
$$


In order to estimate $J_{2}$ we apply Weyl's inequality. The latter shows that when $X$ is sufficiently large, and $|f(\beta ; X)| \geq X^{3 / 4+\eta}$, then there exist $a \in \mathbb{Z}$ and $q \in \mathbb{N}$ with $(a, q)=1$,

$$
|q \beta-a| \leq \frac{1}{10} X^{-9 / 4} \quad \text { and } \quad q \leq \frac{1}{10} X^{3 / 4}
$$

(compare Lemma 1 of Vaughan [17]). Applying the latter conclusion with $\beta=\alpha p^{3}$ and $X=P / p$, we find that whenever $\left|f\left(\alpha p^{3} ; P / p\right)\right| \geq Q^{3 / 4+\eta}$, then there exist $a \in \mathbb{Z}$ and $q \in \mathbb{N}$ with $(a, q)=1$,

$$
\left|\alpha p^{3} q-a\right| \leq Q^{-9 / 4} \text { and } q \leq Q^{3 / 4}
$$

But $p^{3} q \leq 8 Y^{3} Q^{3 / 4}<P Y^{3}$ and $Q^{9 / 4} \geq P^{2} Y^{-3}$, so that $\alpha \notin \mathfrak{m}$. Hence, uniformly for $\alpha \in \mathfrak{m}$ we have

$$
\sum_{p}\left|f\left(\alpha p^{3} ; P / p\right)\right|^{2} \ll Y Q^{3 / 2+\varepsilon} .
$$

On applying Cauchy's inequality to (5.7), we therefore deduce that

$$
\begin{aligned}
J_{2} & \leq \int_{\mathfrak{m}}\left(\sum_{p}\left|f\left(\alpha p^{3} ; P / p\right)\right|^{2}\right)^{2}\left(\sum_{p}\left|h\left(\alpha p^{3}\right)\right|^{2}\right)^{2} d \alpha \\
& \ll Y Q^{3 / 2+\varepsilon} \int_{0}^{1}\left(\sum_{p}\left|f\left(\alpha p^{3} ; P / p\right)\right|^{2}\right)\left(\sum_{p}\left|h\left(\alpha p^{3}\right)\right|^{2}\right)^{2} d \alpha .
\end{aligned}
$$

A second application of Cauchy's inequality now yields the estimate

$$
J_{2} \ll Y^{2} Q^{3 / 2+\varepsilon} \int_{0}^{1}\left(\sum_{p_{1}}\left|f\left(\alpha p_{1}^{3} ; P / p_{1}\right)\right|^{2}\right)\left(\sum_{p_{2}}\left|h\left(\alpha p_{2}^{3}\right)\right|^{4}\right) d \alpha .
$$

On considering the underlying diophantine equation, Lemma 3 shows that the integral on the right hand side of (5.11) is $O\left(Y Q^{3+\xi+\varepsilon}\right)$, so that on combining (5.9) and (5.11), we arrive at the estimate

$$
I(Y) \ll Y^{\frac{5}{2}} P^{\frac{3}{2}+\varepsilon} Q^{\frac{9}{4}+\frac{\xi}{2}} \ll P^{4} Q^{-1 / 8} .
$$

A modest calculation reveals that this estimate is stronger than that claimed in the conclusion of Theorem 3 in the range $P^{1 / 8} \leq Y \leq P^{1 / 7}$ under consideration. 
Suppose next that $I_{2} \leq I_{1}$. Then in view of (2.8) and (5.3), by applying Cauchy's inequality to (5.4) we obtain the estimate

$$
I(Y) \leq 2 I(Y)^{1 / 2}\left(\int_{\mathfrak{m}}\left|\sum_{p} f_{p}(\alpha) h\left(\alpha p^{3}\right)\right|^{2}|g(\alpha)|^{3} d \alpha\right)^{1 / 2} .
$$

But as a consequence of Hölder's inequality,

$$
\left|\sum_{p} f_{p}(\alpha) h\left(\alpha p^{3}\right)\right|^{2} \leq Y\left(\sum_{p}\left|f_{p}(\alpha)^{2} h\left(\alpha p^{3}\right)^{5}\right|\right)^{2 / 5}\left(\sum_{p}\left|f_{p}(\alpha)\right|^{2}\right)^{3 / 5}
$$

whence by a second application of Hölder's inequality, we deduce from (5.12) that

$$
I(Y) \ll Y I_{3}^{2 / 5} I_{4}^{3 / 5}
$$

where

(5.14) $I_{3}=\sum_{p} \int_{\mathfrak{m}}\left|f_{p}(\alpha)^{2} h\left(\alpha p^{3}\right)^{5}\right| d \alpha \quad$ and $\quad I_{4}=\sum_{p} \int_{\mathfrak{m}}\left|f_{p}(\alpha)^{2} g(\alpha)^{5}\right| d \alpha$.

Note that $I_{3}$ is the integral estimated in (4.10) above, and hence we may concentrate on estimating $I_{4}$. The latter integral strongly resembles $I(Y)$, but the exponential sum $f_{p}(\alpha)$ occurs in place of $f(\alpha)$. One expects that the additional implicit coprimality condition does not significantly alter the magnitude of the integral, and hence one should have that $I_{4} \ll Y I(Y)$. Unfortunately, the respective integrals are restricted to the minor arcs $\mathfrak{m}$, and so the required estimates are not immediate from a consideration of the underlying diophantine equations. However, we can invert the process used at the beginning of this proof in order to circumvent such difficulties. Thus, by (5.1) we have

$$
\left|f_{p}(\alpha)\right|^{2} \leq 2\left(|f(\alpha)|^{2}+\left|f\left(\alpha p^{3} ; P / p\right)\right|^{2}\right)
$$

and so by (5.14) and (2.8) we have

$$
I_{4} \ll Y I(Y)+I_{5}
$$

where

$$
I_{5}=\int_{\mathfrak{m}} \sum_{p}\left|f\left(\alpha p^{3} ; P / p\right)^{2} g(\alpha)^{5}\right| d \alpha
$$


In circumstances where $I_{5} \leq Y I(Y)$, it follows from (5.13), (5.15) and (4.10) that

$$
I(Y) \ll Y^{4} I_{3} \ll Y^{\frac{3}{2}} P^{\frac{3}{2}} Q^{\frac{5}{2}+\gamma+\varepsilon},
$$

whence the conclusion of Theorem 3 is immediate. We may therefore suppose that $I_{5}>Y I(Y)$, and then one obtains from (5.13), (5.15) and (4.10) the estimate

$$
I(Y) \ll P^{\frac{3}{5}} Q^{1+\frac{2}{5} \gamma+\varepsilon} I_{5}^{\frac{3}{5}} .
$$

But in view of (5.10), an application of Schwarz's inequality to (5.16) yields

$$
I_{5} \ll Y Q^{3 / 2+\varepsilon}\left(\int_{0}^{1}|g(\alpha)|^{4} d \alpha\right)^{1 / 2}\left(\int_{0}^{1}|g(\alpha)|^{6} d \alpha\right)^{1 / 2} .
$$

On applying Hua's Lemma (see Lemma 2.5 of Vaughan [20]) to estimate the first integral on the right hand side of (5.18), and (5.8) to bound the second, we find that

$$
I_{5} \ll Y Q^{3 / 2+\varepsilon}\left(P^{2+\varepsilon}\right)^{1 / 2}\left(P^{3+\varepsilon} Y^{2}\right)^{1 / 2} \ll P^{4+\varepsilon} Y^{1 / 2} .
$$

Thus we deduce from (5.17) that

$$
I(Y) \ll P^{3} Q^{1+\frac{2}{5} \gamma+\varepsilon} Y^{\frac{3}{10}}
$$

and again a modest computation shows that this estimate is stronger than that claimed in the conclusion of Theorem 3 in the range $P^{1 / 8} \leq Y \leq P^{1 / 7}$ under consideration. This completes the proof of Theorem 3.

\section{Three Cubes and a Sixth Power}

\section{$\S 6$. An outline}

The main objective of this chapter is the proof of Theorem 1 . We begin by examining another mean value which relates to a diophantine equation involving two cubes and ten sixth powers, such forming the subject of $\S 7$. We are then in a position to describe the generating function fundamental to our proof of Theorem 1 , this being the theme of $\S 8$, where we also prepare for an application of the Hardy-Littlewood method. A rather thin and narrow choice of major arcs is made in $\S 8$, and we are able to provide a lower bound for the contribution from these arcs immediately. This turns out to be routine, even though the singular series causes some mild complications. 
There remains the problem of handling the minor arcs. If the latter were as thin as those occurring in the statement of Theorem 3, then their contribution would be easily controlled by combining the conclusions of Theorem 3 and Lemma 5 below through the medium of Schwarz's inequality. However, the sizes of the major and minor arcs are not immediately compatible, and thus we are left with some intermediate arcs of which to dispose in $\S 9$. Here we work a little harder than is necessary for the application at hand, this treatment being of some independent interest (part V of Brüdern, Kawada and Wooley [7]). Theorem 1 is an immediate consequence of the conclusion of $\S 8$ combined with the main result (Theorem 4) of $\S 9$.

\section{$\S 7$. Another auxiliary equation}

In this section we apply an iterative method to estimate a mean value corresponding to two cubes and ten sixth powers. By and large we continue to use the notation introduced in the previous chapter. In this context, we recall the definition of $f(\alpha)=f(\alpha ; P)$ from $(2.1)$, and when $\mathcal{B} \subseteq[1, \sqrt{P}] \cap \mathbb{Z}$ we define the exponential sum $b(\alpha)=b(\alpha ; \mathcal{B})$ by

$$
b(\alpha ; \mathcal{B})=\sum_{y \in \mathcal{B}} e\left(\alpha y^{6}\right) .
$$

Finally, we define the mean value $K=K(P ; \mathcal{B})$ by

$$
K(P ; \mathcal{B})=\int_{0}^{1}\left|f(\alpha ; P)^{2} b(\alpha ; \mathcal{B})^{10}\right| d \alpha .
$$

We may now formulate the main result of this section.

LEMma 5. There is a positive number $\eta_{0}$ such that whenever $R \leq P^{\eta_{0}}$ and $\mathcal{B} \subseteq \mathcal{A}(\sqrt{P}, R)$, one has

$$
K(P ; \mathcal{B}) \ll P^{4.191218}
$$

Proof. We make use of the "new iterative method", the framework provided by Wooley [23] being a suitable version for sums of mixed powers. Let $K_{0}$ denote the number of integral solutions of the equation

$$
x_{1}^{3}-x_{2}^{3}=\sum_{j=1}^{5}\left(y_{j}^{6}-z_{j}^{6}\right)
$$


with $1 \leq x_{i} \leq 2 P(i=1,2)$ and $y_{j}, z_{j} \in \mathcal{A}\left(P^{1 / 13}, R\right)(1 \leq j \leq 5)$. Also, define the mean value $K_{1}$ by

$$
K_{1}=\int_{0}^{1}\left|F(\alpha ; P)^{2} b(\alpha ; \mathcal{B})^{8}\right| d \alpha .
$$

Finally, let $K_{2}$ denote the number of integral solutions of the equation

$$
x_{1}^{3}-x_{2}^{3}=m^{6} \sum_{j=1}^{5}\left(y_{j}^{6}-z_{j}^{6}\right),
$$

with

(7.6) $1 \leq x_{i} \leq 2 P(i=1,2), \quad P^{1 / 13}<m \leq P^{1 / 13} R, \quad x_{1} \equiv x_{2}\left(\bmod m^{6}\right)$,

$$
y_{j}, z_{j} \in \mathcal{A}(Z, R) \quad(1 \leq j \leq 5),
$$

where for the sake of concision we write $Z=P^{11 / 26}$. Then on applying Lemma 2.2 of Wooley [23] with $k=6, s=5, \theta=\frac{1}{13}, Q=\sqrt{P}$, we deduce from (7.2) by considering the underlying diophantine equations that

$$
K \ll K_{0}+P^{\frac{15}{26}+\varepsilon} K_{1}+P^{\frac{9}{13}+\varepsilon} R^{9} K_{2} .
$$

One may bound $K_{0}$ easily by observing that there are at most $O\left(P^{10 / 13}\right)$ available choices for $y_{j}, z_{j}(1 \leq j \leq 5)$, and $O(P)$ choices permissible for $x_{2}$. Fixing any one of these choices for the latter variables, the equation (7.3) fixes $x_{1}$. Consequently, we have

$$
K_{0} \ll P^{2}
$$

Before embarking on the analysis of $K_{1}$, it is convenient to record some mean value estimates contained in the appendix of Vaughan and Wooley [22]. Thus, when $s=5,6$ or 8 , it follows from [22] that whenever $\eta$ is a sufficiently small positive number, then one has

$$
\int_{0}^{1}\left|\sum_{z \in \mathcal{A}\left(X, X^{\eta}\right)} e\left(\alpha z^{6}\right)\right|^{2 s} d \alpha \ll X^{\lambda_{s}},
$$

where

$$
\lambda_{5}=5.724697, \quad \lambda_{6}=7.231564, \quad \lambda_{8}=10.560413 .
$$


On considering the underlying diophantine equations, it follows from (7.10) that

$$
\int_{0}^{1}|b(\alpha)|^{12} d \alpha \ll P^{\frac{1}{2} \lambda_{6}}
$$

Moreover, on applying Schwarz's inequality in combination with Hua's lemma (see Lemma 2.5 of Vaughan [20]), one obtains

$$
\int_{0}^{1}|F(\alpha)|^{6} d \alpha \leq\left(\int_{0}^{1}|F(\alpha)|^{4} d \alpha\right)^{1 / 2}\left(\int_{0}^{1}|F(\alpha)|^{8} d \alpha\right)^{1 / 2} \ll P^{\frac{7}{2}+\varepsilon} .
$$

Consequently, by Hölder's inequality it follows from (7.4) that

$$
K_{1} \leq\left(\int_{0}^{1}|F(\alpha)|^{6} d \alpha\right)^{1 / 3}\left(\int_{0}^{1}|b(\alpha)|^{12} d \alpha\right)^{2 / 3} \ll P^{\frac{7}{6}+\frac{1}{3} \lambda_{6}+\varepsilon} .
$$

In view of (7.11), we may therefore conclude in this case that

$$
P^{\frac{15}{26}+\varepsilon} K_{1} \ll P^{4.16} .
$$

Finally, we estimate $K_{2}$. This is more elaborate, and entails another application of the Hardy-Littlewood method. When

$$
P^{1 / 13} \leq M \leq P^{1 / 13} R
$$

we take $T(M)$ to be the number of solutions $m, \mathbf{x}, \mathbf{y}, \mathbf{z}$ of (7.5) counted by $K_{2}$ in which $M<m \leq 2 M$. Then a dyadic dissection argument yields the upper bound

$$
K_{2} \ll(\log P) T(M),
$$

for some $M$ satisfying (7.13). Given a solution $m, \mathbf{x}, \mathbf{y}, \mathbf{z}$ of (7.5) counted by $T(M)$, we write

$$
h=\left(x_{1}-x_{2}\right) m^{-6} \quad \text { and } \quad z=x_{1}+x_{2} .
$$

Then in view of (7.6) one has that $h$ is an integer satisfying $|h| \leq H$, where $H$ now denotes $2 P M^{-6}$. Write

$$
F_{1}(\alpha)=\sum_{1 \leq h \leq H} \sum_{M<m \leq 2 M} \sum_{1 \leq z \leq 4 P} e\left(\alpha h\left(3 z^{2}+h^{2} m^{12}\right)\right)
$$

and

$$
t(\alpha)=\sum_{y \in \mathcal{A}(Z, R)} e\left(\alpha y^{6}\right)
$$


On substituting from (7.15) into (7.5), isolating the diagonal contribution, and considering the underlying diophantine equation, we obtain the estimate

$$
T(M) \ll P M \int_{0}^{1}|t(4 \alpha)|^{10} d \alpha+\int_{0}^{1}\left|F_{1}(\alpha) t(4 \alpha)^{10}\right| d \alpha .
$$

We now apply the Hardy-Littlewood method to estimate the second integral on the right hand side of (7.18). Let $\mathfrak{K}$ denote the union of the major arcs

$$
\mathfrak{K}(q, a)=\left\{\alpha \in[0,1]:|q \alpha-a| \leq(H P)^{-1}\right\}
$$

with $0 \leq a \leq q \leq P$ and $(a, q)=1$, and let $\mathfrak{k}=[0,1] \backslash \mathfrak{K}$. Bounds for $F_{1}(\alpha)$ are obtained through the use of Cauchy's inequality in the form

$$
\left|F_{1}(\alpha)\right|^{2} \leq D(\alpha) E(\alpha)
$$

where

$$
D(\alpha)=\sum_{1 \leq h \leq H}\left|\sum_{1 \leq z \leq 4 P} e\left(3 \alpha h z^{2}\right)\right|^{2}
$$

and

$$
E(\alpha)=\sum_{1 \leq h \leq H}\left|\sum_{M<m \leq 2 M} e\left(\alpha h^{3} m^{12}\right)\right|^{2} .
$$

As in Lemma 3.1 of Vaughan [19], one has

$$
\sup _{\alpha \in \mathfrak{k}} D(\alpha) \ll H P^{1+\varepsilon}
$$

and also, when $\alpha \in \mathfrak{K}(q, a) \subseteq \mathfrak{K}$,

$$
D(\alpha) \ll H P^{2+\varepsilon}\left(q+H P^{2}|q \alpha-a|\right)^{-1} .
$$

In order to estimate $E(\alpha)$ we may argue as in the proof of Lemma 3.4 of Vaughan [19]. For $M \leq P^{1 / 12}$ one finds, mutatis mutandis, that whenever $a \in \mathbb{Z}$ and $q \in \mathbb{N}$ satisfy

$$
|q \alpha-a| \leq\left(H^{3} M^{6}\right)^{-1} \quad \text { and } \quad 1 \leq q \leq H^{3} M^{6}
$$

then one has

$$
E(\alpha) \ll P^{\varepsilon} H M+P^{\varepsilon} H M^{2}\left(q+H P^{2}|q \alpha-a|\right)^{-1 / 6}
$$


(this estimate occurs also as a special case of Ford $[9,(3.10)])$. On recalling (7.13), we deduce from (7.20)-(7.23) that

$$
\sup _{\alpha \in \mathfrak{k}}\left|F_{1}(\alpha)\right| \ll\left(H P^{1+\varepsilon}\right)^{1 / 2}\left(P^{\varepsilon} H M\right)^{1 / 2} \ll P^{\varepsilon}(P M)^{1 / 2} H
$$

Consequently, it follows from (7.18) and (7.10) that

$$
T(M) \ll P^{1+\varepsilon} M Z^{\lambda_{5}}+\int_{\mathfrak{K}}\left|F_{1}(\alpha) t(4 \alpha)^{10}\right| d \alpha .
$$

We estimate the integral remaining in (7.24) through the use of the auxiliary mean value $J_{0}$, which we define by

$$
J_{0}=\int_{\mathfrak{K}}\left|F_{1}(\alpha)^{2} t(4 \alpha)^{4}\right| d \alpha .
$$

Plainly, by (7.17) one has

$$
|t(4 \alpha)|^{4}=\sum_{l \in \mathbb{Z}} \psi(l) e(l \alpha)
$$

where $\psi(l)$ denotes the number of solutions of the equation

$$
4\left(z_{1}^{6}+z_{2}^{6}-z_{3}^{6}-z_{4}^{6}\right)=l,
$$

with $z_{i} \in \mathcal{A}(Z, R)(1 \leq i \leq 4)$. Observe that by Hua's Lemma (see Lemma 2.5 of Vaughan [20]) and an elementary counting argument, one has

$$
\psi(0) \ll Z^{2+\varepsilon} \quad \text { and } \quad \sum_{l \in \mathbb{Z}} \psi(l)=t(0)^{4} \ll Z^{4} .
$$

Next let $\mathfrak{L}$ denote the union of the major arcs $\mathfrak{K}(q, a)$ (defined in (7.19)) with $0 \leq a \leq q \leq M^{6}$ and $(a, q)=1$. Then by (7.23) we have

$$
\sup _{\alpha \in \mathfrak{K} \backslash \mathfrak{L}} E(\alpha) \ll P^{\varepsilon} H M
$$

whence by (7.20) and (7.22), whenever $\alpha \in \mathfrak{K}(q, a) \subseteq \mathfrak{K} \backslash \mathfrak{L}$, one has

$$
\left|F_{1}(\alpha)\right|^{2} \ll P^{2+\varepsilon} H^{2} M\left(q+H P^{2}|q \alpha-a|\right)^{-1} .
$$

By employing (7.26) and (7.27) within Lemma 2 of Brüdern [3], we obtain the estimate $(7.28) \int_{\mathfrak{K} \backslash \mathfrak{L}}\left|F_{1}(\alpha)^{2} t(4 \alpha)^{4}\right| d \alpha \ll P^{\varepsilon} M H\left(P Z^{2+\varepsilon}+Z^{4}\right) \ll P^{1+\varepsilon} M H Z^{2}$. 
When $\alpha \in \mathfrak{K}(q, a) \subseteq \mathfrak{K}$, we may use the trivial bound $E(\alpha) \ll H M^{2}$ in combination with $(7.22)$ to deduce that

$$
\left|F_{1}(\alpha)\right|^{2} \ll P^{\varepsilon}(P H M)^{2}\left(q+H P^{2}|q \alpha-a|\right)^{-1} .
$$

Thus, again by Lemma 2 of Brüdern [3], we have (7.29) $\int_{\mathfrak{L}}\left|F_{1}(\alpha)^{2} t(4 \alpha)^{4}\right| d \alpha \ll P^{\varepsilon} H M^{2}\left(M^{6} Z^{2+\varepsilon}+Z^{4}\right) \ll P^{\varepsilon} H M^{2} Z^{4}$.

Finally, on collecting together (7.25), (7.28) and (7.29), we obtain the upper bound

$$
J_{0} \ll P^{1+\varepsilon} M H Z^{2} .
$$

Equipped with the above bound for $J_{0}$, we now apply Schwarz's inequality to the integral on the right hand side of (7.24), so that by (7.10), (7.11) and (7.30) we have

$$
\begin{aligned}
\int_{\mathfrak{K}}\left|F_{1}(\alpha) t(4 \alpha)^{10}\right| d \alpha & \ll J_{0}^{1 / 2}\left(\int_{0}^{1}|t(\alpha)|^{16} d \alpha\right)^{1 / 2} \\
& \ll P^{\varepsilon}(P M H)^{1 / 2} Z^{1+\frac{1}{2} \lambda_{8}} \ll P^{1-\tau} M Z^{\lambda_{5}}
\end{aligned}
$$

where $\tau>0.03$. It therefore follows from (7.11), (7.14) and (7.24) that

$$
P^{\frac{9}{13}+\varepsilon} R^{9} K_{2} \ll P^{\frac{23}{13}+\frac{11}{26} \lambda_{5}+\varepsilon} R^{10} \ll P^{4.191218},
$$

whenever $\eta_{0}$ is a sufficiently small positive number. On substituting (7.9), (7.12) and (7.31) into (7.8), the proof of the lemma is completed.

\section{$\S 8$. The proof of Theorem 1: the generating function}

We now prepare the final components of the machinery required in our proof of Theorem 1. Let $N$ be a large real number, and write

$$
P=\left[\left(\frac{1}{4} N\right)^{1 / 3}\right] \text { and } \tau=10^{-20} \text {. }
$$

Also, define the set $\mathcal{Y}$ by

$$
\mathcal{Y}=\left\{Y \in\left[P^{1 / 8}, P^{1 / 8+\tau}\right]: Y=2^{l} P^{1 / 8} \text { and } l \in \mathbb{N}\right\} .
$$

We note for future reference that $\operatorname{card}(\mathcal{Y}) \asymp \log P$. Let $\eta$ be a positive number sufficiently small in the context of Theorem 3 and Lemma 5, and write $R=P^{\eta}$. Recalling (2.1), (2.7) and (7.1), we then write

$$
f(\alpha)=f(\alpha ; P), \quad g(\alpha ; Y)=g(\alpha ; Y ; \mathcal{C}(P / Y, R)), \quad b(\alpha)=b(\alpha ; \mathcal{C}(\sqrt{P}, R)) .
$$


Also, we define the exponential sum $G(\alpha)$ by

$$
G(\alpha)=\sum_{Y \in \mathcal{Y}} g(\alpha ; Y)
$$

Finally, define

$$
\nu(n)=\int_{0}^{1} f(\alpha) G(\alpha)^{2} b(\alpha) e(-n \alpha) d \alpha .
$$

Thus, by orthogonality it is apparent that $\nu(n)$ is a lower bound for the number of integral solutions of the equation

$$
n=x^{3}+\left(p_{1} y_{1}\right)^{3}+\left(p_{2} y_{2}\right)^{3}+z^{6},
$$

with

$$
\begin{gathered}
P<x \leq 2 P, \quad z \in \mathcal{C}(\sqrt{P}, R) \\
P^{1 / 8} \leq p_{i} \leq 2 P^{1 / 8+\tau}, \quad y_{i} \in \mathcal{C}\left(2 P / p_{i}, R\right) \quad(i=1,2) .
\end{gathered}
$$

On recalling the definition of $\nu_{6}(n)$ from the introduction, it follows in particular that $\nu_{6}(n) \geq \nu(n)$. In order to establish Theorem 1 , it therefore suffices to show that there is a positive number $\sigma$ such that for all but $O\left(N^{1-\sigma}\right)$ of the integers $n \in[N, 2 N]$, one has $\nu(n) \gg \mathfrak{S}(n) \sqrt{P}$, the desired conclusion following by summing over dyadic intervals.

We analyse the integral in (8.5) by means of the Hardy-Littlewood method. Write $W=R^{1 / 50}$, and let $\mathfrak{P}$ denote the union of the major arcs

$$
\mathfrak{P}(q, a)=\left\{\alpha \in[0,1]:|\alpha-a / q| \leq W P^{-3}\right\}
$$

with $0 \leq a \leq q \leq W$ and $(a, q)=1$, and let $\mathfrak{p}=[0,1] \backslash \mathfrak{P}$. We require an asymptotic formula for the integrand in (8.5), at least when $\alpha \in \mathfrak{P}$. In this context, when $k=3$ or 6 , we define

$$
S_{k}(q, a)=\sum_{r=1}^{q} e\left(a r^{k} / q\right) \quad \text { and } \quad w_{k}(\beta ; L)=\int_{L}^{2 L} e\left(\beta \gamma^{k}\right) d \gamma
$$

We then define

$$
v(\beta)=w_{3}(\beta ; P)
$$

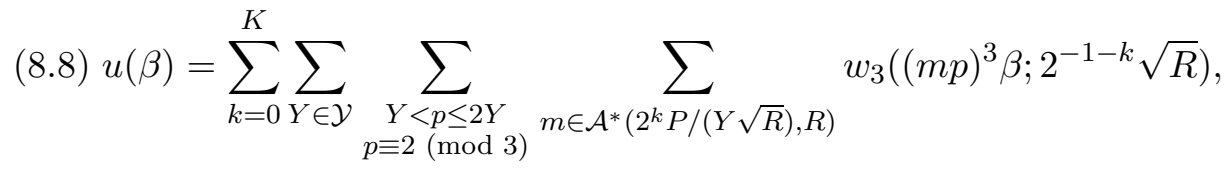




$$
w(\beta)=\sum_{k=0}^{K} \sum_{m \in \mathcal{A}^{*}\left(2^{k} \sqrt{P} / \sqrt{R}, R\right)} w_{6}\left(m^{6} \beta ; 2^{-1-k} \sqrt{R}\right),
$$

in which we write $K=\left[\frac{1}{4} \log R\right]$.

By Theorem 4.1 of Vaughan [20], it follows that whenever $\beta \in \mathbb{R}, a \in \mathbb{Z}$ and $q \in \mathbb{N}$ satisfy $(a, q)=1$, then one has

$$
f(\beta+a / q)-q^{-1} S_{3}(q, a) v(\beta) \ll q^{\frac{1}{2}+\varepsilon}\left(1+P^{3}|\beta|\right)^{\frac{1}{2}} .
$$

Consequently, when $\alpha \in \mathfrak{P}(q, a) \subseteq \mathfrak{P}$, one has the estimate

$$
f(\alpha)=q^{-1} S_{3}(q, a) v(\alpha-a / q)+O\left(W^{1+\varepsilon}\right) .
$$

Observe next that whenever $h=m p$ with $Y<p \leq 2 Y$ for some $Y \in \mathcal{Y}$, and $m \in \mathcal{A}^{*}\left(2^{k} P /(Y \sqrt{R}), R\right)$, then each of the prime divisors of $h$ exceed $R^{1 / 4}>W$. Then whenever $\alpha \in \mathfrak{P}(q, a) \subseteq \mathfrak{P}$, one has $(q, h)=1$, and a change of variables reveals that $S_{3}\left(q, a h^{3}\right)=S_{3}(q, a)$. Thus, in view of (8.3), (8.4) and (8.8), we deduce from Theorem 4.1 of Vaughan [20] that whenever $\alpha \in \mathfrak{P}(q, a) \subseteq \mathfrak{P}$,

$$
G(\alpha)=\sum_{k, Y, p, m} \sum_{2^{-1-k} \sqrt{R}<l \leq 2^{-k} \sqrt{R}} e\left(\alpha(p m l)^{3}\right)
$$

is equal to

$$
\sum_{k, Y, p, m}\left(q^{-1} S_{3}\left(q, a(p m)^{3}\right) w_{3}\left((p m)^{3}(\alpha-a / q) ; 2^{-1-k} \sqrt{R}\right)+O\left(W^{1+\varepsilon}\right)\right),
$$

where the summations are over $0 \leq k \leq K, Y \in \mathcal{Y}, Y<p \leq 2 Y$ with $p \equiv 2(\bmod 3)$, and $m \in \mathcal{A}^{*}\left(2^{k} P /(Y \sqrt{R}), R\right)$. Consequently,

$$
G(\alpha)=q^{-1} S_{3}(q, a) u(\alpha-a / q)+O\left(P W^{-4}\right) .
$$

Similarly, though more simply, it follows from (8.9) that whenever $\alpha \in$ $\mathfrak{P}(q, a) \subseteq \mathfrak{P}$, one has

$$
b(\alpha)=q^{-1} S_{6}(q, a) w(\alpha-a / q)+O\left(\sqrt{P} W^{-4}\right) .
$$

Since the measure of $\mathfrak{P}$ is plainly $O\left(W^{3} P^{-3}\right)$, it follows from (8.10)-(8.12) by means of a modest calculation that

$$
\int_{\mathfrak{P}} f(\alpha) G(\alpha)^{2} b(\alpha) e(-n \alpha) d \alpha=J_{0}(n) \sum_{1 \leq q \leq W} A(q, n)+O\left(\sqrt{P} W^{-1}\right)
$$


where

$$
\begin{gathered}
J_{0}(n)=\int_{-W P^{-3}}^{W P^{-3}} v(\beta) u(\beta)^{2} w(\beta) e(-n \beta) d \beta, \\
A(q, n)=q^{-4} \sum_{\substack{a=1 \\
(a, q)=1}}^{q} S_{3}(q, a)^{3} S_{6}(q, a) e(-n a / q) .
\end{gathered}
$$

On recalling (8.6), a partial integration shows that for each $\beta \in \mathbb{R}$ one has

$$
w_{k}(\beta ; L) \ll L\left(1+L^{k}|\beta|\right)^{-1} .
$$

Consequently, for each $\beta \in \mathbb{R}$,

$$
v(\beta) \ll P\left(1+P^{3}|\beta|\right)^{-1} .
$$

By analogy, one would routinely expect that one also has

$$
u(\beta) \ll P\left(1+P^{3}|\beta|\right)^{-1 / 3},
$$

but a verification of this bound requires some care. By Theorem 3 on p.400 of Tenenbaum [16], one has the bound

$$
\operatorname{card}\left(\mathcal{A}^{*}(Q, R)\right) \ll Q(\log R)^{-1}
$$

uniformly for $Q \geq \sqrt{R} \geq 2$. But for $0<Q<\sqrt{R}$, the set $\mathcal{A}^{*}(Q, R)$ is empty, and therefore the aforementioned bound remains valid for all $Q>0$. For $\theta>0$ and $Q \geq T \geq 1$, we now estimate the sum

$$
U_{0}(\theta)=\sum_{m \in \mathcal{A}^{*}(Q / T, R)} \frac{T}{1+T^{3} m^{3} \theta} .
$$

When $\theta \leq Q^{-3}$, we have

$$
U_{0}(\theta) \leq T \operatorname{card}\left(\mathcal{A}^{*}(Q / T, R)\right) \ll Q(\log R)^{-1} .
$$

When $\theta>Q^{-3}$, meanwhile, we find that

$$
U_{0}(\theta) \leq T \operatorname{card}\left(\mathcal{A}^{*}\left(M_{0}, R\right)\right)+\sum_{\substack{m \in \mathcal{A}^{*}(Q / T, R) \\ m>M_{0}}} T^{-2} \theta^{-1} m^{-3}
$$


where $M_{0}=T^{-1} \theta^{-1 / 3}$. But whenever $M_{0}<M<Q / T$, we have

$$
\sum_{\substack{m \in \mathcal{A}^{*}(Q / T, R) \\ M<m \leq 2 M}} m^{-3} \ll M^{-3} \operatorname{card}\left(\mathcal{A}^{*}(2 M, R)\right) .
$$

Now take $M=2^{l} M_{0}$ and sum over $l$ to conclude that for each $\theta>0$, we have

$$
U_{0}(\theta) \ll Q(\log R)^{-1}\left(1+Q^{3} \theta\right)^{-1 / 3} .
$$

Recalling (8.8) together with the routine bound for $w_{3}(\beta ; L)$ recorded above, we now observe that

$$
u(\beta) \ll \sum_{Y \in \mathcal{Y}} \sum_{Y<p \leq 2 Y} \sum_{k=0}^{K} \sum_{m \in \mathcal{A}^{*}\left(Q / T_{k}, R\right)} \frac{T_{k}}{1+T_{k}^{3} m^{3} p^{3}|\beta|},
$$

where $Q=P / Y$ and $T_{k}=2^{-k} \sqrt{R}$. With the bound for $U_{0}\left(p^{3}|\beta|\right)$ just obtained, and recalling that $K \ll \log R$, we finally deduce that

$$
u(\beta) \ll \sum_{Y \in \mathcal{Y}} \sum_{Y<p \leq 2 Y} Q\left(1+Q^{3} p^{3}|\beta|\right)^{-1 / 3} .
$$

The expected upper bound (8.17) follows by an elementary summation. A similar argument yields

$$
w(\beta) \ll \sqrt{P}\left(1+P^{3}|\beta|\right)^{-1 / 6} .
$$

Write

$$
J(n)=\int_{-\infty}^{\infty} v(\beta) u(\beta)^{2} w(\beta) e(-\beta n) d \beta .
$$

Then by substituting (8.16)-(8.18) into (8.14), we find that the integral $J(n)$ is absolutely convergent, and moreover that

(8.20) $J_{0}(n)-J(n) \ll \int_{W P^{-3}}^{\infty} P^{7 / 2}\left(1+P^{3} \beta\right)^{-11 / 6} d \beta \ll \sqrt{P} W^{-5 / 6}$.

Furthermore, in like manner,

$$
J(n) \ll \sqrt{P} .
$$

In order to bound from below the singular integral $J(n)$, we require an estimate for the cardinality of the set $\mathcal{A}^{*}(Q, R)$. Here we make use of the 
work of Friedlander [10] (more recent work of Saias [15] is also available). Suppose that $A, B$ and $C$ are fixed real numbers with $B>A \geq 1$ and $C>0$. Let $Q$ and $R$ be large real numbers satisfying $R^{A} \leq Q \leq R^{B}$. Then as an immediate consequence of Theorem 1 of Friedlander [10], one has the bounds

(8.22) $\frac{C Q}{\log R} \ll_{A, B} \operatorname{card}\left(\mathcal{A}^{*}((1+C) Q, R)\right)-\operatorname{card}\left(\mathcal{A}^{*}(Q, R)\right) \ll_{A, B} \frac{C Q}{\log R}$.

Next we observe that by making a change of variable in (8.6), for each positive number $\zeta$ one has

$$
w_{k}\left(\zeta^{k} \beta ; L\right)=\zeta^{-1} \int_{\zeta L}^{2 \zeta L} e\left(\beta \gamma^{k}\right) d \gamma
$$

Define $J^{*}(n)=J^{*}(n ; \mathbf{Z})$ by

$$
J^{*}(n ; \mathbf{Z})=\int_{-\infty}^{\infty} \int_{\mathfrak{B}(\mathbf{Z})} e\left(\beta\left(\gamma_{1}^{3}+\gamma_{2}^{3}+\gamma_{3}^{3}+\gamma_{4}^{6}-n\right)\right) d \boldsymbol{\gamma} d \beta,
$$

where

$$
\mathfrak{B}(\mathbf{Z})=\left[Z_{0}, 2 Z_{0}\right] \times\left[Z_{1}, 2 Z_{1}\right] \times\left[Z_{2}, 2 Z_{2}\right] \times\left[Z_{3}, 2 Z_{3}\right] .
$$

Then with $\mathbf{Z}=\mathbf{Z}(\mathbf{m}, \mathbf{p}, \mathbf{k})$ defined by

$$
Z_{0}=P, \quad Z_{i}=2^{-1-k_{i}} m_{i} p_{i} \sqrt{R} \quad(i=1,2), \quad Z_{3}=2^{-1-k_{3}} m_{3} \sqrt{R},
$$

one finds from (8.19) and (8.7)-(8.9) that

$$
J(n)=\sum_{\mathbf{k}, \mathbf{Y}, \mathbf{p}, \mathbf{m}}\left(m_{1} m_{2} m_{3} p_{1} p_{2}\right)^{-1} J^{*}(n ; \mathbf{Z})
$$

where the summation is over

(8.23) $0 \leq k_{i} \leq K \quad(1 \leq i \leq 3), \quad Y_{1}, Y_{2} \in \mathcal{Y}, \quad m_{3} \in \mathcal{A}^{*}\left(2^{k_{3}} \sqrt{P} / \sqrt{R}, R\right)$,

$$
\begin{aligned}
& Y_{j}<p_{j} \leq 2 Y_{j}, \quad p_{j} \equiv 2(\bmod 3), \\
& m_{j} \in \mathcal{A}^{*}\left(2^{k_{j}} P /\left(Y_{j} \sqrt{R}\right), R\right) \quad(j=1,2) .
\end{aligned}
$$

Whenever

$$
2^{1 / 2} Y_{i} \leq p_{i} \leq 2^{3 / 4} Y_{i} \quad(i=1,2)
$$

(8.26) $m_{j} \geq 2^{k_{j}-\frac{1}{4}} P /\left(Y_{j} \sqrt{R}\right) \quad(j=1,2) \quad$ and $\quad m_{3} \geq 2^{k_{3}-\frac{1}{4}} \sqrt{P} / \sqrt{R}$, 
one has that

$$
[P, 2 P] \times\left[2^{-1 / 4} P, 2^{1 / 4} P\right]^{2} \times\left[2^{-2 / 3} \sqrt{P}, 2^{-1 / 3} \sqrt{P}\right] \subseteq \mathfrak{B}(\mathbf{Z})
$$

Thus an application of Fourier's integral formula rapidly establishes that

$$
J(n) \gg \sum_{\mathbf{k}, \mathbf{Y}, \mathbf{p}, \mathbf{m}}\left(m_{1} m_{2} m_{3} p_{1} p_{2}\right)^{-1} \sqrt{P},
$$

where the summations are subject to (8.23)-(8.26). In view of (8.22) and an elementary prime number estimate, therefore, one obtains the lower bound

$$
J(n) \gg \sqrt{P} \sum_{\mathbf{k}, \mathbf{Y}}(\log R)^{-3}\left(\log Y_{1}\right)^{-1}\left(\log Y_{2}\right)^{-1} \gg \sqrt{P} .
$$

In combination with (8.21), we thus conclude that

$$
\sqrt{P} \ll J(n) \ll \sqrt{P} .
$$

It remains to complete the singular series. As this is not quite as straightforward as one might expect, we provide a moderate level of detail. We begin with a simple observation. Suppose that $t$ is a natural number with $(t, q)=1$. Then when $k=3$ or 6 , one may make a change of variable in (8.6) to deduce that $S_{k}\left(q, a t^{k}\right)=S_{k}(q, a)$. On making use of this observation within (8.15), we deduce that

$$
\text { (8.28) } \begin{aligned}
A(q, n) & =\frac{1}{\phi(q)} \sum_{\substack{t=1 \\
(t, q)=1}}^{q} q^{-4} \sum_{\substack{a=1 \\
(a, q)=1}}^{q} S_{3}(q, a)^{3} S_{6}(q, a) e\left(-a t^{6} n / q\right) \\
& =\frac{1}{q^{4} \phi(q)} \sum_{\substack{a=1 \\
(a, q)=1}}^{q} S_{3}(q, a)^{3} S_{6}(q, a) S_{6}^{*}(q,-a n)
\end{aligned}
$$

where we write

$$
S_{6}^{*}(q, b)=\sum_{\substack{t=1 \\(t, q)=1}}^{q} e\left(b t^{6} / q\right) .
$$

But by Lemma 1.2 of Hua [11], whenever $(b, q)=1$ one has $S_{6}^{*}(q, b) \ll$ $q^{1 / 2+\varepsilon}$, whence by the multiplicativity of the latter exponential sum, when $(a, q)=1$ one has
$(8.29)$

$$
S_{6}^{*}(q,-a n) \ll q^{1 / 2+\varepsilon}(q, n)^{1 / 2} .
$$


Also, when $(a, q)=1$, Lemmata $4.3-4.5$ of Vaughan [20] yield the estimate

$$
q^{-1} S_{k}(q, a) \ll \kappa_{k}(q)
$$

where $\kappa_{k}(q)$ is a multiplicative function defined on prime powers by

$$
\kappa_{k}\left(p^{l k+1}\right)=k p^{-l-\frac{1}{2}}, \quad \kappa_{k}\left(p^{l k+j}\right)=p^{-l-1} \quad(l \geq 0,2 \leq j \leq k) .
$$

On combining (8.28)-(8.30), it follows that

$$
A(q, n) \ll q^{1 / 2+\varepsilon} \kappa_{3}(q)^{3} \kappa_{6}(q)(q, n)^{1 / 2} .
$$

Recalling (8.31) and making use of the multiplicativity of the underlying generating functions, it is readily confirmed that the series

$$
\sum_{q=1}^{\infty} q^{7 / 12} \kappa_{3}(q)^{3} \kappa_{6}(q)
$$

converges absolutely. Since $(q, n) \leq n$, we deduce from (8.32) that the singular series

$$
\mathfrak{S}(n)=\sum_{q=1}^{\infty} A(q, n)
$$

also converges absolutely for every natural number $n$. Next write

$$
\omega_{p}(n)=\sum_{h=0}^{\infty} A\left(p^{h}, n\right) .
$$

Then by (8.31) and (8.32), it follows that for each prime $p$ one has

$$
\omega_{p}(n)-1 \ll p^{\varepsilon-3 / 2}(p, n)^{1 / 2} .
$$

Since $A(q, n)$ is multiplicative, we may rewrite $\mathfrak{S}(n)$ as a product,

$$
\mathfrak{S}(n)=\prod_{p} \omega_{p}(n)
$$

and by (8.35) this product is again absolutely convergent. Next we observe that the argument underlying the proof of Lemma 2.12 of Vaughan [20] shows that when $h \geq 1$, one has

$$
\sum_{l=0}^{h} A\left(p^{l}, n\right)=p^{-3 h} \Omega\left(p^{h}, n\right),
$$


where $\Omega\left(p^{h}, n\right)$ denotes the number of incongruent solutions of the congruence

$$
x_{1}^{3}+x_{2}^{3}+x_{3}^{3}+y^{6} \equiv n\left(\bmod p^{h}\right) .
$$

In particular, therefore, it follows from (8.34) that $\omega_{p}(n)$ is real and nonnegative. When $p \neq 3$ and $h=1$, it follows from the Cauchy-Davenport Theorem (see Lemma 2.14 of Vaughan [20]) that the congruence (8.36) is soluble with $p \nmid x_{1}$. A modest computation reveals that when $p^{h}=9$ and $n \not \equiv 5(\bmod 9)$, then the congruence $(8.36)$ is again soluble with $3 \nmid x_{1}$. The methods of $\S 2.6$ of Vaughan [20], in combination with (8.35), therefore show that for any sufficiently large but fixed positive number $C$, one has

$$
\mathfrak{S}(n) \gg \prod_{\substack{p \mid n \\ p>C}} \omega_{p}(n) .
$$

We now refine (8.35) in those cases where $p \mid n$. A careful use of (8.32) shows that in the latter circumstances, one has

$$
\omega_{p}(n)=1+A(p, n)+A\left(p^{3}, n\right)+A\left(p^{6}, n\right)+O\left(p^{\varepsilon-3 / 2}\right) .
$$

By (8.32), we also have $A\left(p^{6}, n\right) \ll p^{\varepsilon-3 / 2}$ unless $p^{6} \mid n$, and similarly, one has $A\left(p^{3}, n\right) \ll p^{\varepsilon-3 / 2}$ unless $p^{3} \mid n$. But in the exceptional cases, we observe that when $k=3$ or 6 , it follows from Lemma 4.4 of Vaughan [20] that whenever $p \nmid a, p>3$ and $2 \leq l \leq k$, one has $S_{k}\left(p^{l}, a\right)=p^{l-1}$. Then we deduce from (8.28) that whenever $p^{3} \mid n$ one has

$$
A\left(p^{3}, n\right)=\left(p^{3}\right)^{-4} \phi\left(p^{3}\right)\left(p^{2}\right)^{4}=\frac{1}{p}-\frac{1}{p^{2}},
$$

and similarly that whenever $p^{6} \mid n$, one has

$$
A\left(p^{6}, n\right)=\left(p^{6}\right)^{-4} \phi\left(p^{6}\right)\left(p^{4}\right)^{3} p^{5}=\frac{1}{p}-\frac{1}{p^{2}} \text {. }
$$

Thus, in all cases where $A\left(p^{3}, n\right)$ or $A\left(p^{6}, n\right)$ cannot be absorbed into the error term, these numbers are real and positive. Since $1+A(p, n)$ is also real, we infer that the inequality

$$
\omega_{p}(n) \geq 1+A(p, n)+O\left(p^{\varepsilon-3 / 2}\right)
$$


holds for all primes $p \mid n$. From (8.37) we now deduce that

$$
\mathfrak{S}(n) \gg \prod_{\substack{p \mid n \\ p>C}}(1+A(p, n)) .
$$

Furthermore, when $p \mid n$, a multiplicative change of variables shows that given a quadratic non-residue $\nu$ modulo $p$, one has

$$
\begin{aligned}
A(p, n)= & p^{-4} \sum_{a=1}^{p-1} S_{3}\left(p, a \nu^{3}\right)^{3} S_{6}\left(p, a \nu^{3}\right) \\
& =p^{-4} \sum_{a=1}^{p-1} S_{3}(p, a)^{3} S_{6}\left(p, a \nu^{3}\right) .
\end{aligned}
$$

But writing $(\dot{\bar{p}})$ for the quadratic residue symbol modulo $p$, one has

$$
S_{6}(p, a)=\sum_{s=1}^{p}\left(1+\left(\frac{s}{p}\right)\right) e\left(\frac{a s^{3}}{p}\right)
$$

and thus

$$
\begin{aligned}
S_{6}\left(p, a \nu^{3}\right) & =\sum_{s=1}^{p}\left(1+\left(\frac{s}{p}\right)\right) e\left(\frac{a(\nu s)^{3}}{p}\right) \\
& =\sum_{t=1}^{p}\left(1+\left(\frac{\nu t}{p}\right)\right) e\left(\frac{a t^{3}}{p}\right) \\
& =\sum_{t=1}^{p}\left(1-\left(\frac{t}{p}\right)\right) e\left(\frac{a t^{3}}{p}\right) .
\end{aligned}
$$

An inspection of the conjugate reveals that $S_{3}(p, a)$ is always real, and thus it follows from $(8.39)-(8.41)$ that

$$
\text { (8.42) } \begin{aligned}
A(p, n) & =\frac{1}{2}\left(p^{-4} \sum_{a=1}^{p-1} S_{3}(p, a)^{3} S_{6}(p, a)+p^{-4} \sum_{a=1}^{p-1} S_{3}(p, a)^{3} S_{6}\left(p, a \nu^{3}\right)\right) \\
& =p^{-4} \sum_{a=1}^{p-1} S_{3}(p, a)^{4}=p^{-4} \sum_{a=1}^{p-1}\left|S_{3}(p, a)\right|^{4} \geq 0 .
\end{aligned}
$$


At last, on substituting (8.42) into (8.38), we conclude that

$$
\mathfrak{S}(n) \gg 1
$$

uniformly in $n \not \equiv 5(\bmod 9)$, as claimed in the introduction.

In order to complete our discussion of the major arc contribution, we return to the estimate (8.13). Using $d(q)$ to denote the number of divisors of $q$, and recalling the absolute convergence of the sum (8.33), we deduce from (8.32) that

$$
\sum_{N \leq n \leq 2 N} \sum_{q>W}|A(q, n)| \ll N \sum_{q>W} d(q) q^{\frac{1}{2}+\varepsilon} \kappa_{3}(q)^{3} \kappa_{6}(q) \ll N W^{-1 / 15} .
$$

It follows that the number of integers $n$ with $N \leq n \leq 2 N$ for which

$$
\sum_{q>W}|A(q, n)|>W^{-\tau}
$$

is $O\left(N W^{-1 / 20}\right)$. On recalling (8.43), we therefore find that for all but $O\left(N W^{-1 / 20}\right)$ of the integers $n \not \equiv 5(\bmod 9)$ with $N \leq n \leq 2 N$, we have

$$
\sum_{1 \leq q \leq W} A(q, n) \gg \mathfrak{S}(n) \gg 1
$$

Combining the latter conclusion with (8.13), (8.20) and (8.27), we may summarise the conclusions of this section as follows.

Lemma 6. For all but $O\left(N R^{-1 / 1000}\right)$ of the integers $n$ with $n \not \equiv 5$ $(\bmod 9)$ and $N \leq n \leq 2 N$, one has

$$
\int_{\mathfrak{P}} f(\alpha) G(\alpha)^{2} b(\alpha) e(-n \alpha) d \alpha \gg \mathfrak{S}(n) \sqrt{P} .
$$

Moreover, the singular series satisfies $\mathfrak{S}(n) \gg 1$ uniformly in $n \not \equiv 5(\bmod 9)$.

\section{$\S$ 9. A minor arc estimate}

In this section we complement the lower bound recorded in Lemma 6 with an upper bound for the contribution arising from the minor arcs. As a by-product we obtain a mean square estimate which has already found application in our recent joint work with Kawada [7]. We therefore highlight this result as another theorem. In stating this, we continue to use notation 
familiar from previous sections, but modify the notation defined in (8.3) by writing

$$
g(\alpha ; Y ; \mathcal{B})=g(\alpha ; Y ; \mathcal{B}(P / Y, R)), \quad b(\alpha ; \mathcal{B})=b(\alpha ; \mathcal{B}(\sqrt{P}, R)),
$$

where it is understood that $\mathcal{B}$ denotes one of $\mathcal{A}$ and $\mathcal{C}$, and by writing, further,

$$
G(\alpha ; \mathcal{B})=\sum_{Y \in \mathcal{Y}} g(\alpha ; Y ; \mathcal{B})
$$

THEOREM 4. Let $X$ be a real number with $1 \leq X \leq P^{10 / 7}$, and write $\mathfrak{m}(X)$ for the set of real numbers $\alpha \in[0,1]$ such that whenever $q$ is a natural number with $\|q \alpha\| \leq X P^{-3}$, one has $q>X$. Then with $\tau=10^{-20}$, and with $\mathcal{B}$ equal to either $\mathcal{A}$ or $\mathcal{C}$, we have

$$
\int_{\mathfrak{m}(X)}\left|f(\alpha) G(\alpha ; \mathcal{B})^{2} b(\alpha ; \mathcal{B})\right|^{2} d \alpha \ll P^{4} X^{-\tau}
$$

Before proving Theorem 4, we pause to knock off the proof of Theorem 1 , which at this stage is easily accomplished. We take $\mathcal{B}=\mathcal{C}$, and suppress explicit mention of this set in the exponential sums, for the sake of concision. Then by appealing to (9.2) with $X=R^{1 / 50}$ and observing that $\mathfrak{p} \subseteq \mathfrak{m}(X)$, we deduce from Bessel's inequality that

$$
\begin{aligned}
\sum_{n \in \mathbb{N}}\left|\int_{\mathfrak{p}} f(\alpha) G(\alpha)^{2} b(\alpha) e(-n \alpha) d \alpha\right|^{2} & \leq \int_{\mathfrak{m}(X)}\left|f(\alpha) G(\alpha)^{2} b(\alpha)\right|^{2} d \alpha \\
& \ll P^{4} X^{-\tau} .
\end{aligned}
$$

We deduce that there are at most $O\left(P^{3} X^{-\tau / 2}\right)$ integers $n$ for which the inequality

$$
\left|\int_{\mathfrak{p}} f(\alpha) G(\alpha)^{2} b(\alpha) e(-n \alpha) d \alpha\right|>\sqrt{P} X^{-\tau / 4}
$$

holds. On recalling (8.5) and applying Lemma 6, we find that for all but $O\left(N R^{-\frac{\tau}{100}}\right)$ of the integers $n$ with $n \not \equiv 5(\bmod 9)$ and $N \leq n \leq 2 N$, one has

$$
\begin{aligned}
\nu(n) & =\int_{\mathfrak{P}} f(\alpha) G(\alpha)^{2} b(\alpha) e(-n \alpha) d \alpha+\int_{\mathfrak{p}} f(\alpha) G(\alpha)^{2} b(\alpha) e(-n \alpha) d \alpha \\
& \gg \mathfrak{S}(n) \sqrt{P}+O\left(\sqrt{P} X^{-\tau / 4}\right) \gg \mathfrak{S}(n) n^{1 / 6} .
\end{aligned}
$$

The conclusion of Theorem 1 follows immediately on summing over dyadic intervals. 
The proof of Theorem 4. Write $X_{0}=P^{\frac{11}{8}+3 \tau}$, and again suppress explicit mention of sets in exponential sums. Then in view of (8.2), for any $Y \in \mathcal{Y}$ one has $\mathfrak{m}\left(X_{0}\right) \subseteq \mathfrak{m}$, where $\mathfrak{m}$ is the set of minor arcs defined in the statement of Theorem 3. Then by (9.1), Hölder's inequality and (2.9), one has the upper bound

$$
\int_{\mathfrak{m}\left(X_{0}\right)}\left|f(\alpha)^{2} G(\alpha)^{5}\right| d \alpha \ll(\log P)^{4} \sum_{Y \in \mathcal{Y}} I(Y) \ll P^{3.95211} .
$$

With a second appeal to Hölder's inequality, we infer from Lemma 5 that

$$
\begin{aligned}
\int_{\mathfrak{m}\left(X_{0}\right)} \mid f(\alpha) & \left.G(\alpha)^{2} b(\alpha)\right|^{2} d \alpha \\
& \leq\left(\int_{\mathfrak{m}\left(X_{0}\right)}\left|f(\alpha)^{2} G(\alpha)^{5}\right| d \alpha\right)^{4 / 5}\left(\int_{0}^{1}\left|f(\alpha)^{2} b(\alpha)^{10}\right| d \alpha\right)^{1 / 5} \\
& \ll P^{4-2 \tau}
\end{aligned}
$$

since

$$
\frac{4}{5}(3.95211)+\frac{1}{5}(4.191218)<3.99994 .
$$

When $X \geq X_{0}$, the proof of Theorem 4 is complete.

Suppose next that $X<X_{0}$. Denote by $\mathfrak{M}(\Xi)$ the union of the intervals

$$
\mathfrak{M}(q, a ; \Xi)=\left\{\alpha \in[0,1]:|q \alpha-a| \leq \Xi P^{-3}\right\}
$$

with $0 \leq a \leq q \leq \Xi$ and $(a, q)=1$, and then write

$$
\mathfrak{M}_{0}=\mathfrak{M}\left(X_{0}\right), \quad \mathfrak{M}_{1}=\mathfrak{M}\left(P^{15 / 16}\right), \quad \mathfrak{M}_{2}=\mathfrak{M}\left(P^{14 \tau}\right)
$$

We dispose of the contribution of the arcs $\mathfrak{M}_{0} \backslash \mathfrak{M}_{1}$ by combining Theorem 4.1 and the methods of Lemma 6.3 of Vaughan [20] to establish the estimate

$$
\sup _{\alpha \in \mathfrak{M}_{0} \backslash \mathfrak{M}_{1}}|f(\alpha)| \ll P\left(P^{\frac{15}{16}}\right)^{-\frac{1}{3}}+X_{0}^{\frac{1}{2}+\varepsilon} \ll P^{\frac{11}{16}+2 \tau} .
$$

By (9.1) and Hölder's inequality, we deduce from Lemma 2 that

$$
\int_{0}^{1}\left|f(\alpha)^{2} G(\alpha)^{4}\right| d \alpha \ll P^{\frac{13}{4}+3 \tau}
$$


and on considering the underlying diophantine equations, this estimate also yields

$$
\int_{0}^{1}|G(\alpha)|^{6} d \alpha \ll P^{\frac{13}{4}+3 \tau} .
$$

On combining (9.4)-(9.6) with Lemma 5 by means of yet another application of Hölder's inequality, we discover that

$$
\begin{aligned}
\int_{\mathfrak{M}_{0} \backslash \mathfrak{M}_{1}} \mid f(\alpha) & \left.G(\alpha)^{2} b(\alpha)\right|^{2} d \alpha \\
\ll & \left.\sup _{\alpha \in \mathfrak{M}_{0} \backslash \mathfrak{M}_{1}}|f(\alpha)|\right)^{\frac{4}{5}}\left(\int_{0}^{1}\left|f(\alpha)^{2} G(\alpha)^{4}\right| d \alpha\right)^{\frac{2}{5}} \\
& \quad \times\left(\int_{0}^{1}|G(\alpha)|^{6} d \alpha\right)^{\frac{2}{5}}\left(\int_{0}^{1}\left|f(\alpha)^{2} b(\alpha)^{10}\right| d \alpha\right)^{\frac{1}{5}} \\
& \ll\left(P^{\frac{11}{16}+2 \tau}\right)^{\frac{4}{5}}\left(P^{\frac{13}{4}+3 \tau}\right)^{\frac{4}{5}}\left(P^{4.191218}\right)^{\frac{1}{5}} \ll P^{3.99} .
\end{aligned}
$$

Another pruning procedure, which is applicable for $X \leq P^{15 / 16}$, has been made available only very recently in work of the authors [8]. We note that by (8.31) one has $\kappa_{3}(q) \geq q^{-1 / 2}$ for all natural numbers $q$. Then by Theorem 4.1 and the methods of Lemma 6.2 of Vaughan [20], together with the estimate (8.30) above, whenever $\alpha \in \mathfrak{M}(q, a ; X) \subseteq \mathfrak{M}_{1}$ one has

$$
|f(\alpha)| \ll \kappa_{3}(q) P\left(1+P^{3}|\alpha-a / q|\right)^{-1 / 2} .
$$

Since we may write

$$
G(\alpha)=\sum_{x \in \mathcal{S}} e\left(\alpha x^{3}\right),
$$

where $\mathcal{S}$ is some subset of $[1,2 P]$, it follows from Lemma 3.1 of Brüdern and Wooley [8] that for $1 \leq \Xi \leq P^{15 / 16}$ one has

$$
\int_{\mathfrak{M}(\Xi)}|f(\alpha) G(\alpha)|^{2} d \alpha \ll P \Xi^{\varepsilon} .
$$

It is now straightforward to complete the proof. When $\mathcal{B}=\mathcal{A}$ and $\Xi \leq P^{15 / 16}$, the estimate

$$
\sup _{\alpha \in \mathfrak{m}(\Xi)}|b(\alpha)|^{2} \ll P^{1-3 \tau}+P \Xi^{\varepsilon-\frac{1}{6}}
$$

is an immediate consequence of Lemmata 7.2 and 8.5 of Vaughan and Wooley [21]. When $\mathcal{B}=\mathcal{C}$ and $1 \leq \Xi \leq R^{1 / 50}$, the estimate (9.9) follows by 
combining (8.12), (8.18), (8.30) and (8.31). Meanwhile, when $\mathcal{B}=\mathcal{C}$ and $R^{1 / 50}<\Xi \leq P^{15 / 16}$, one may apply the method of the proof of Lemma 7.2 of Vaughan and Wooley [21] to again deduce that (9.9) holds. One has merely to note that in the proof of [21, Lemma 7.2], the fact that the exponential sum is over the full set $\mathcal{A}(P, R)$ is irrelevant, and indeed the method is equally applicable when $\mathcal{A}(P, R)$ is replaced by any subset thereof. Since $\mathcal{C}(Q, R) \subseteq \mathcal{A}(Q, R)$, it is apparent that such is the case in the present application, and thus our earlier assertion is justified. On combining (9.8) and (9.9) with the trivial estimate $G(\alpha)=O(P)$, we deduce that

$$
\begin{aligned}
\int_{\mathfrak{M}_{1} \backslash \mathfrak{M}_{2}}\left|f(\alpha) G(\alpha)^{2} b(\alpha)\right|^{2} d \alpha \\
\quad \ll P^{2}\left(\sup _{\alpha \in \mathfrak{m}\left(P^{14 \tau}\right)}|b(\alpha)|^{2}\right) \int_{\mathfrak{M}_{1}}|f(\alpha) G(\alpha)|^{2} d \alpha \\
\ll P^{4-2 \tau} .
\end{aligned}
$$

When $X \geq P^{14 \tau}$, the proof of the theorem is completed by collecting together (9.3), (9.7) and (9.10). When $X \leq P^{14 \tau}$ we may argue similarly. For $X \leq \Xi \leq P^{14 \tau}$, it follows from (9.8) and (9.9) that

$$
\begin{aligned}
\int_{\mathfrak{M}(2 \Xi) \backslash \mathfrak{M}(\Xi)}\left|f(\alpha) G(\alpha)^{2} b(\alpha)\right|^{2} d \alpha \\
\quad \ll P^{2}\left(\sup _{\alpha \in \mathfrak{m}(\Xi)}|b(\alpha)|^{2}\right) \int_{\mathfrak{M}(2 \Xi)}|f(\alpha) G(\alpha)|^{2} d \alpha \\
\quad \ll P^{4-2 \tau}+P^{4} \Xi^{-1 / 7}
\end{aligned}
$$

and thus the proof of Theorem 4 is readily completed by summing over dyadic intervals.

\section{REFERENCES}

[1] T. Breyer, Über die Summe von sechs Kuben und zwei sechsten Potenzen, Diplomarbeit, Universität Göttingen, 1996.

[2] J. Brüdern, Iterationsmethoden in der additiven Zahlentheorie, Dissertation, Göttingen, 1988.

[3] _ A problem in additive number theory, Math. Proc. Cambridge Philos. Soc., 103 (1988), 27-33.

[4] On Waring's problem for cubes and biquadrates. II, Math. Proc. Cambridge Philos. Soc., 104 (1988), 199-206. 
[5] (1991), 229-256.

[6] — A note on cubic exponential sums, Sém. Théorie des Nombres, Paris, 1990-1991 (S. David, ed.), Progr. Math. 108, Birkhäuser Boston, Boston MA (1993), pp. 23-34.

[7] J. Brüdern, K. Kawada and T. D. Wooley, Additive representation in thin sequences, IV: lower bound methods, Quart. J. Math. Oxford (2) (in press); V: mixed problems of Waring type, Math. Scand. (to appear).

[8] J. Brüdern and T. D. Wooley, On Waring's problem for cubes and smooth Weyl sums, Proc. London Math. Soc. (3), 82 (2001), 89-109.

[9] K. B. Ford, The representation of numbers as sums of unlike powers. II, J. Amer. Math. Soc., 9 (1996), 919-940.

[10] J. B. Friedlander, Integers free from large and small primes, Proc. London Math. Soc. (3), 33 (1976), 565-576.

[11] L.-K. Hua, On the representation of numbers as the sums of the powers of primes, Math. Z., 44 (1938), 335-346.

[12] K. Kawada, On the sum of four cubes, Mathematika, 43 (1996), 323-348.

[13] Ming Gao Lu, On Waring's problem for cubes and higher powers, Chin. Sci. Bull., 37 (1992), 1414-1416.

[14] - On Waring's problem for cubes and fifth power, Sci. China Ser. A, 36 (1993), 641-662.

[15] E. Saias, Entiers sans grand ni petit facteur premier. I, Acta Arith., 61 (1992), $347-374$.

[16] G. Tenenbaum, Introduction to analytic and probabilistic number theory. Cambridge Studies in Advanced Mathematics, 46, Cambridge University Press, Cambridge, 1995.

[17] R. C. Vaughan, On Waring's problem for cubes, J. Reine Angew. Math., 365 (1986), $122-170$.

[18] _ On Waring's problem for cubes II, J. London Math. Soc. (2), 39 (1989), 205-218.

[19] — A new iterative method in Waring's problem, Acta Math., 162 (1989), 1-71.

[20] , The Hardy-Littlewood method, 2nd edition, Cambridge University Press, Cambridge, 1997.

[21] R. C. Vaughan and T. D. Wooley, On Waring's problem: some refinements, Proc. London Math. Soc. (3), 63 (1991), 35-68.

$[22] \longrightarrow$, Further improvements in Waring's problem, Acta Math., 174 (1995), $147-240$.

[23] T. D. Wooley, Large improvements in Waring's problem, Ann. of Math. (2), 135 (1992), 131-164.

[24] — Breaking classical convexity in Waring's problem: sums of cubes and quasi-diagonal behaviour, Inventiones Math., 122 (1995), 421-451. 
Jörg Brüdern

Mathematisches Institut A

Universität Stuttgart

Postfach 801140

D-70511 Stuttgart

Germany

bruedern@mathematik. uni-stuttgart.de

Trevor D. Wooley

Department of Mathematics

University of Michigan, East Hall

525 East University Avenue

Ann Arbor, Michigan 48109-1109

U.S.A.

wooley@math. Isa.umich.edu 